\title{
Sparsity based off-grid blind sensor calibration
}

\author{
Sedat Camlica ${ }^{\mathrm{a}, *}$, Imam Samil Yetik $^{\mathrm{b}}$, Orhan Arikan $^{\mathrm{c}}$ \\ a Aselsan A.S., Ankara, 06370 Turkey \\ ${ }^{\mathrm{b}}$ Department of Electrical and Electronics Engineering, TOBB University of Economics and Technology, Ankara 06560, Turkey \\ ${ }^{\mathrm{c}}$ Department of Electrical and Electronics Engineering, Bilkent University, Ankara 06800, Turkey
}

\section{A R T I C L E I N F O}

\section{Article history:}

Available online 25 October 2018

\section{Keywords:}

Blind calibration

Sparse

Compressive sensing

Off-grid

Direction finding

Frequency estimation

\begin{abstract}
A B S T R A C T
Compressive Sensing (CS) based techniques generally discretize the signal space and assume that the signal has a sparse support restricted on the discretized grid points. This restriction of representing the signal on a discretized grid results in the off-grid problem which causes performance degradation in the reconstruction of signals. Sensor calibration is another issue which can cause performance degradation if not properly addressed. Calibration aims to reduce the disruptive effects of the phase and the gain biases. In this paper, a CS based blind calibration technique is proposed for the reconstruction of multiple off-grid signals. The proposed technique is capable of estimating the off-grid signals and correcting the gain and the phase biases due to insufficient calibration simultaneously. It is applied to off-grid frequency estimation and direction finding applications using blind calibration. Extensive simulation analyses are performed for both applications. Results show that the proposed technique has superior reconstruction performance.
\end{abstract}

(C) 2018 Elsevier Inc. All rights reserved.

\section{Introduction}

Sparsity based Compressive Sensing (CS) methods have gained high importance in recent years, since they play a central role in many practical application areas. CS methods make it possible to operate under Nyquist rate if the signal is sparse in a perfectly known transform domain satisfying certain constraints [1-3]. Because of very promising properties and possible advantages, CS based techniques have received considerable attention in radar, sensor and civilian applications [4,5].

In radar and sensor systems, calibration is an important issue, which directly affects the system performance thus must be addressed carefully. Aging and/or environmental effects such as temperature changes introduce system errors making calibration capability an unavoidable need for a given system. Furthermore, imperfect knowledge of the system parameters may cause calibration errors [6-8]. Guided calibration solutions can be used to achieve desired performance in which a known training signal is used. However, such a technique increases the cost and complexity of the underlying system. In systems such as sensor and/or radar systems, phased array antennas, MIMO systems, blind calibration

\footnotetext{
* Corresponding author.

E-mail addresses: scamlica@aselsan.com.tr (S. Camlica), syetik@etu.edu.tr (I.S. Yetik), oarikan@ee.bilkent.edu.tr (O. Arikan).
}

is an alternative technique that estimates the signal first and performs the calibration using the estimated signal. It is beneficial in terms of reducing maintenance costs and/or complexity of the system; as well as avoiding the need for a training signal.

In the literature, there are various methods for blind sensor calibration. In [9], sparsity based convex optimization methods for blind sensor calibration are studied. Signal reconstruction and calibration are performed simultaneously. Phase only, gain only and joint phase and gain blind calibration methods are proposed [9]. In [6], blind calibration of sensor networks is performed using ordinary sensor measurements. In [7], sensor networks are calibrated using signal sources with known locations. Sensor positions, phase and gain errors and mutual coupling between sensors are estimated. A sparsity based conjugate gradient algorithm is used for blind sensor calibration in [10]. In [11], sparsity based blind calibration of sensor networks is studied using total least squares approach. Sparsity based blind sensor calibration is also studied in [12] for phased array sensors. In [13], a moments based blind calibration method is studied for mobile sensor networks. It is assumed that the sensors are located in the same region and observed signals have almost identical statistics which is exploited for calibration. In [14], a non-convex blind calibration method is presented for linear random sensing models with positive noncomplex gain errors. A similar compressive sensing based greedy blind calibration method for positive non-complex gain errors is also presented in [15]. 
As mentioned, there are sparsity based methods which are capable of jointly estimating the signal and correcting the phase and the gain errors. However, another source of error that invalidates the exact sparsity assumptions and affect the reconstruction performance of sparsity based techniques is the basis mismatch. These techniques discretize the signal space and assume that the signal is sparse on the discretized grid. Due to continuous nature of the signals, representing the signal on a discretized grid results in the off-grid problem which causes performance degradation. In the CS literature the off-grid problem also exists in frequency estimation [16], angle of arrival (AOA) estimation [16], delay-Doppler imaging [17], and SAR imaging [18]. There are various types of solutions to off-grid target problem in CS [18,17,19-22].

The current sparsity based blind sensor calibration methods, as to best of our knowledge, do not deal with the off-grid signals. Therefore, development of such a method, which is capable of jointly estimating the signal and handling the phase and the gain calibration and off-grid phase errors, is mandatory.

In [18], our group developed a sparsity based Spotlight mode Synthetic Aperture Radar imaging and autofocus method for offgrid sparse scenes. From a mathematical point of view, the proposed method [18] estimates a two dimensional off-grid signal and performs phase error calibration simultaneously.

In this work, our method in [18] is expanded to blind sensor calibration with multiple snapshots and a novel sparsity based blind sensor calibration method for off-grid signals is developed. We also apply the proposed method to two different problems and show the effectiveness for these example application areas. The proposed method handles the off-grid problem using a gradient search based optimization algorithm while being capable of estimating and calibrating phase and gain errors as well as offgrid perturbations simultaneously for multiple measurements as a novel contribution. The current blind calibration techniques do not take care of off-grid signal errors, and the proposed technique, as to best of our knowledge, is the first technique that does blind sensor calibration under both phase, gain and off-grid signal errors jointly. The proposed off-grid blind sensor calibration algorithm (Perturbed Calibration Orthogonal Matching Pursuit, PCOMP) is first derived for the frequency estimation problem and corresponding simulation analysis is given in detail. Then, PC-OMP algorithm is adopted to off-grid direction finding problem with certain required variations and simulation analysis is also given for this case. Although the proposed algorithm is applied to the frequency estimation and direction finding problems, it can be easily adopted to any other application concerning array processing as long as a convenient measurement model can be constructed. For example, the proposed technique can be applied to phased array radar systems, Electronic Warfare reconnaissance systems such as frequency spectrum sensing, direction finding, target localization. Simulation results show that the proposed methods have superior performance in terms of the calculated metrics.

The organization of this paper is as follows: the off-grid signal model with calibration errors is given in Section 2. The detailed derivation of the proposed method for the general case is given in Section 3 and simulations are given in Section 4. Off-grid direction finding and blind calibration method is explained in Section 5 with simulations for this case and conclusions are drawn in Section 6 .

The notation throughout the text is as follows: lowercase bold letters (i.e. $\mathbf{x}$ ) indicates column vectors, uppercase bold letters (i.e. G) indicates matrices, $\|$.$\| indicates l_{2}$ norm and |.| indicates absolute value.

\section{Signal model}

A sensor array is considered in which individual sensors samples the received signal at the same time, then the measurements are processed together. It is assumed that each sensor has calibration errors (i.e. phase and gain errors) which are constant over time and independent from other sensors. For such an array system, let $y_{i}(n)$ be the signal measured by $n$th sensor at time instance $i$. For $K$ sources, $y_{i}(n)$ can be expressed as;

$y_{i}(n)=d_{n} e^{j \phi_{n}} \sum_{k=1}^{K} \alpha_{i, k} g_{n}\left(f_{i, k}^{o}\right)+w_{i}$,

where $d_{n}$ and $\phi_{n}$ are the gain and phase calibration errors respectively, $\alpha_{i, k}$ are the complex signal gains, $g_{n}($.$) is the equivalent$ basis vector, and $f_{i, k}^{o}$ is the signal parameter.

For $N_{y}$ number of sensors, writing the measurements in vector form yields $\mathbf{y}_{i}=\left[y_{i}(1) y_{i}(2) \ldots y_{i}\left(N_{y}\right)\right]^{T}$. The on-grid based techniques assumes that the signal parameters relies exactly on a grid, and using the grid parameters they populate the relevant basis vectors which (by assumption) spans the signal space completely. Thus a measurement model matrix can be constructed. Using the vector form notation and measurement model matrix, the blind sensor calibration signal model for the on-grid case can be expressed as;

$\left[\begin{array}{llll}\mathbf{y}_{1} & \mathbf{y}_{2} & \ldots & \mathbf{y}_{D}\end{array}\right]=\boldsymbol{\Phi G}\left[\begin{array}{llll}\mathbf{x}_{1} & \mathbf{x}_{2} & \ldots & \mathbf{x}_{D}\end{array}\right]+\mathbf{w}$,

where $D$ is the number of measurements; $\mathbf{x}_{i}$ and $\mathbf{y}_{i}$ are the desired signal to be estimated and corresponding measurement vectors, whose dimensions are $N_{x} \times 1$ and $N_{y} \times 1$, respectively. $N_{y}$ is the number of sensors and $\mathbf{w}$ is the additive zero-mean white noise $N_{y} \times D$ matrix. Let $\boldsymbol{\Psi}$ be the measurement model matrix, then $\mathbf{G}=\mathbf{\Psi A}$, where $\mathbf{A}$ is the basis matrix on which the signal $\mathbf{x}_{i}$ is sparse, and $\boldsymbol{\Phi}$ is $N_{y} \times N_{y}$ diagonal matrix whose entries contain calibration errors. Then, $\boldsymbol{\Phi}$ is defined as:

$\boldsymbol{\Phi}=\left[\begin{array}{cccc}d_{1} e^{j \phi_{1}} & & & \\ & d_{2} e^{j \phi_{2}} & & \\ & & \ddots & \\ & & & d_{N_{y}} e^{j \phi_{N_{y}}}\end{array}\right]$.

$\boldsymbol{\Phi}$ being a diagonal matrix makes the calibration errors independent among different sensors. In blind calibration, the error parameters $d_{n}$ and $\phi_{n}$ are to be jointly estimated and corrected in the proposed method PC-OMP.

For the off-grid signal case, the signal parameters $f_{i, k}^{o}$ are assumed to be rely on anywhere in the signal space rather than a known grid locations. Thus, a predefined measurement model matrix can not be constructed, instead it must also be estimated along with other desired signal parameters. In the off-grid case $\mathbf{y}_{i}$ is expressed as:

$\mathbf{y}_{i}=\boldsymbol{\Phi} \sum_{k=1}^{K} \alpha_{i, k} \mathbf{g}\left(f_{i, k}^{o}\right)+\mathbf{w}_{i}$,

where $\alpha_{i, k}$ are the complex signal gains, $\mathbf{g}($.) the equivalent basis vector used to represent the signal and defined as $\mathbf{g}\left(f_{k}\right)=\boldsymbol{\Psi} \mathbf{a}\left(f_{k}\right)$. Here, $f_{i, k}^{o}$ causes the off-grid problem and is the continuous parameter for $i$ th measurement and $k$ th signal component.

We propose to solve the following optimization problem to estimate the unknown parameters. The cost function is defined as the sum of the squared errors:

$\min _{\alpha_{i, k}, f_{i, k}, \delta f_{i, k}, \phi_{n}, d_{n}} \sum_{i}\left\|\mathbf{y}_{i}-\boldsymbol{\Phi} \boldsymbol{\Psi} \sum_{k=1}^{K} \alpha_{i, k} \mathbf{a}\left(f_{i, k}+\delta f_{i, k}\right)\right\|_{2}^{2}$,

such that: $\sum_{n} d_{n}=c,\left|\delta f_{k}\right|<\Delta / 2,1 \leq i \leq D, 1 \leq n \leq N_{y}$, 
Table 1

Proposed method.

\begin{tabular}{l}
\hline Initialize: $\mathbf{y}^{0}=\mathbf{y}, \mathbf{T}_{0}=\{\}, k=1$ \\
\hline WHILE $k<=K$ \\
OMP Projection and atom selection: \\
FOR $i=1: D$, \\
$j^{*}=\arg \max _{j}\left|\mathbf{g}\left(f_{i, j}\right)^{H} \boldsymbol{r}_{i, k-1}\right|$ \\
$\quad \mathbf{T}_{i, k}=\mathbf{T}_{i, k-1} \cup\left\{f_{j^{*}}\right\}$ \\
Perform blind calibration using (16). \\
END FOR \\
Perform perturbation estimation using (6); \\
FOR $i=1: D$ \\
$\left(\boldsymbol{\alpha}_{i},\left[\delta f_{i, 1}, \ldots, \delta f_{i, k}\right]\right)=\mathbb{S}\left(\mathbf{y}_{i}, \mathbf{T}_{i, k}\right)$ \\
END FOR \\
Repeat blind calibration with updated \\
signal estimates using (16). \\
$k=k+1$ \\
\hline Output: $\left(\boldsymbol{\alpha},\left[\delta f_{i, 1}, \ldots, \delta f_{i, k}\right], \mathbf{T}_{i, k}, \boldsymbol{\Phi}\right)$ \\
\hline
\end{tabular}

where $\Delta$ is the grid cell size, $f_{i, k}$ is the closest grid point, $\delta f_{i, k}$ is the corresponding perturbation which causes off-grid problem, and $c$ is a positive number. There can be found infinite number of solutions for $\mathbf{y}=d \hat{\mathbf{y}}$ in amplitude calibration. To avoid the ambiguity, without loss of generality the constraint of $\sum_{n} d_{n}=c$ is introduced. Simultaneous estimation of the complex signal gains $\left(\alpha_{i, k}\right)$, the signal parameters of $f_{i, k}$ and $\delta f_{i, k}$, and the gain and the phase calibration errors $\left(\phi_{n}, d_{n}\right)$ is the main contribution of this work.

As will be detailed in the next section, the optimization problem in (5) is solved iteratively. The signal parameters $\alpha_{i, k}, f_{i, k}$ and $\delta f_{i, k}$ are estimated first. Then, using these estimated parameters, the phase $\phi_{n}$ and the gain errors $d_{n}$ are estimated and corrected blindly. The iteration is performed until a stopping criterion is met. For instance, a maximum number of iterations or a threshold for the residual error or sparsity level can be used to terminate the iterations.

\section{The proposed method}

The optimization problem in (5) is non-convex and poses a significant challenge to solve directly. Therefore, a sub-optimal iterative method which alternatively solves for the calibration errors and the signal perturbations within a greedy technique is developed. The outer shell of the proposed method is based on Orthogonal Matching Pursuit (OMP) [23] and given in Table 1.

At the $k$ th iteration of the proposed algorithm, for each $\mathbf{y}_{i}$, the selected $k$ grid locations $\mathbf{T}_{i, k}$ and the measurements are input to an inner algorithm abstractly shown as:

$\left(\boldsymbol{\alpha}_{i},\left[\delta f_{i, 1}, \ldots, \delta f_{i, k}\right]\right)=\mathbb{S}\left(\mathbf{y}_{i}, \mathbf{T}_{i, k}\right)$,

with a solver $\mathbb{S}(\cdot)$ which produces a solution to (5) for the selected grid parameters and outputs corresponding complex reflectivities and perturbations. It is important to note that the solver $\mathbb{S}(\cdot)$ is not specifically dependent on OMP and can be integrated into any algorithm that provides a suitable estimation of the initial grid parameters.

Even after an estimate for the $k$-grid positions are provided by the OMP, the optimization problem in (5) is still non-convex and a careful consideration is needed to obtain estimates for the complex reflectivities $\boldsymbol{\alpha}_{i}$, perturbations from the given grid centers $\delta f_{i, k}$ and calibration errors $\phi_{j}$ and $d_{j}$. For this purpose, an iterative optimization approach is developed where each of these parameters is optimized separately while keeping others fixed and this is done iteratively until a convergence criterion is met.
First, the complex amplitude vector $\boldsymbol{\alpha}_{l}$ is found using;

$\boldsymbol{\alpha}_{l}=\arg \min _{\alpha}\left\|\mathbf{y}-\sum_{p=1}^{k} \alpha_{p} \mathbf{g}\left(f_{p, l}\right)\right\|_{2}^{2}$

where $l$ is the iteration index of $\mathbb{S}(\cdot)$ and $f_{p, l}$ is the $f_{p}$ vector in $l$ th iteration. Then, the perturbations are performed, $f_{p, l+1}=$ $f_{p, l}+\delta f_{p, l}$, and $\left[\delta f_{1, l} \ldots \delta f_{k, l}\right]$ is calculated by the following minimization $[17,18]$;

$\min _{\delta f_{p}:\left|\delta f_{p}\right| \leq \Delta_{1} / 2}\left\|\hat{\mathbf{y}}-\sum_{p=1}^{k} \alpha_{p, l} \mathbf{g}\left(f_{p, l}+\delta f_{p}\right)\right\|_{2}^{2}$.

The solution to Eq. (7) is found using the well known least squares method. On the other hand, Eq. (8) is a constrained nonlinear optimization problem. A possible approach to solving this problem is to use a gradient descent technique. For this purpose, the cost function in Eq. (8) can also be linearized around $f_{p, l}$. Further, the expression $\mathbf{g}\left(f_{p, l}+\delta f_{p}\right)$ can be approximated using the first order Taylor series [17,18]. Using first order Taylor series makes it possible to use linear expressions and solve the unknown parameters with a gradient descent approach.

$\mathbf{g}\left(f_{p, l}+\delta f_{p}\right) \approx \mathbf{g}\left(f_{p, l}\right)+\frac{\partial \mathbf{g}}{\partial f_{p, l}} \delta f_{p}$.

Using Eq. (9) and dropping constraints, expression (8) can be rewritten as:

$$
\begin{aligned}
{\left[\delta f_{1, l} \ldots \delta f_{k, l}\right] } & =\underset{\mathbf{u}}{\arg \min } \mathbf{J}_{R}(\mathbf{u}) \\
& =\underset{\mathbf{u}}{\arg \min }\left\|\boldsymbol{r}_{l}-\mathbf{B}_{l} \mathbf{u}\right\|_{2}^{2},
\end{aligned}
$$

where $\boldsymbol{r}_{l}=\hat{\mathbf{y}}-\sum_{p=1}^{k} \alpha_{p, l} \mathbf{g}\left(f_{p, l}\right)$ is the orthogonal residual and $\mathbf{B}_{l} \in$ $\mathcal{C}^{N \times k}$ contains the partial derivatives at the linearization point;

$\mathbf{B}_{l}=\left[\Delta \alpha_{1, l} \frac{\partial \mathbf{g}}{\partial f_{1, l}}, \ldots, \Delta \alpha_{k, l} \frac{\partial \mathbf{g}}{\partial f_{k, l}}\right]$.

Here $\mathbf{u}=\left[\delta f_{1}, \ldots, \delta f_{k}\right]^{T} \in \mathfrak{R}^{k \times 1}$ contains the updates at the $l$ th iteration. When $f_{p, l}$ is calculated, $\mathbf{B}_{l}$ is also updated at each iteration, and the search is performed in the direction of negative gradient. After calculation of the perturbations, the parameters are updated and the updates are limited to single resolution cell of the grid.

For the cost function in Eq. (10), the negative gradient of $J$ at $\mathbf{u}=\mathbf{0}$ is given as $-\left.\nabla_{\mathbf{u}} J(\mathbf{u})\right|_{\mathbf{u}=\mathbf{0}}=\operatorname{Re}\left\{2 \mathbf{B}_{l}^{H} \boldsymbol{r}_{l}\right\}$, which can be used in the following iterative solution of the optimization problem in the Eq. (8):

$\boldsymbol{\alpha}_{l}=\left[\mathbf{g}\left(f_{1, l}\right) \mathbf{g}\left(f_{2, l}\right) \ldots \mathbf{g}\left(f_{k, l}\right)\right]^{\dagger} \mathbf{y}$,

$f_{p, l+1}=f_{p, l}+\mu_{p, l} \operatorname{Re}\left\{\mathbf{B}_{l}^{H} \boldsymbol{r}_{l}\right\}$,

where $\mu_{p, l}$ is the properly chosen step size parameter. The value of the step size can be chosen as a sufficiently small ratio of the initial grid size of the OMP structure given in Table 1 . Note that, setting a too small value will increase the convergence time, and a too large value will increase the estimation error.

In the proposed method, there is no assumption on the measurement sets. Amplitudes and the perturbation parameters are calculated independently for each measurement $\mathbf{y}_{i}$. Then using the calculated parameters, the blind calibration procedure is applied jointly to all measurements. 
Table 2

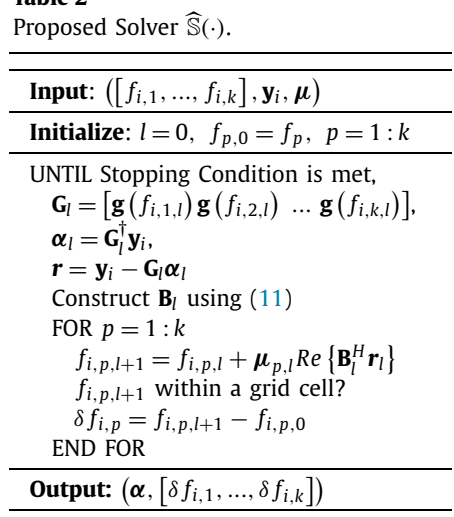

In the process flow of the algorithm given in Table 1 , for each measurement, a grid point is selected using OMP [23] and it is added to the cluster $\mathbf{T}_{i, k}$. Using $\mathbf{T}_{i, k}$ and $\mathbf{y}_{i}$, signal estimation and blind calibration is performed. Then using the calibrated signal and OMP selected grid points, perturbation procedure, given in Table 2, is applied. Finally, using perturbed parameters, blind calibration procedure is re-applied for further improvement in estimation of the phase and the gain errors.

The performance of the iterative method given in Table 1 depends on the choice of stopping criterion for the iterations. When the stopping criterion is not properly chosen, it degrades the performance of the algorithm. To avoid this issue and achieve more clarified performance analysis, the source number, the sparsity level of the signals, is assumed to be known. The iterations are terminated when the estimated signals attain a certain level of sparsity. In the literature, there are methods for estimation of number of sources [24-26]. One of these techniques can be applied prior to the iterations of the proposed technique.

\section{Estimation and correction of the calibration errors}

In this section, estimation of the gain and the phase calibration errors is explained. The phase and the gain error estimation is performed minimizing the cost function $J_{C}\left(d_{n}, \phi_{n}\right)$ which is defined as $l_{2}$ norm of the error between the measurements $\mathbf{y}$ and their estimate $\hat{\mathbf{y}}$ :

$J_{C}\left(d_{n}, \phi_{n}\right)=\underset{d, \phi}{\arg \min }\left\|\mathbf{y}(n)-d e^{j \phi} \hat{\mathbf{y}}(n)\right\|_{2}^{2}$.

Using the calculated parameters in Eq. (4), estimated measurement vector $\hat{\mathbf{y}}_{i}$ is constructed as $\mathbf{y}_{i}=\sum_{k=1}^{K} \alpha_{i, k} \mathbf{g}\left(f_{i, k}^{o}\right)$. Then using these estimates, $\mathbf{y}(n)$ the samples of the $n$th sensor and the $n$th row of the $\hat{\mathbf{y}}$ is constructed and is given as $\hat{\mathbf{y}}(n)=\left[\hat{\mathbf{y}}_{1}(n), \hat{\mathbf{y}}_{2}(n), \ldots, \hat{\mathbf{y}}_{D}(n)\right]$ where $\hat{\mathbf{y}}_{i}(n)$ is the $n$th element of the $\hat{\mathbf{y}}_{i}$.

To estimate the phase and the gain calibration errors, the partial derivatives of the cost function in (13) with respect to $\theta$ and $d$ are formed and equated to zero independently for each parameter.

Then the phase error $\phi_{n}$ is estimated as:

$\hat{\phi}_{n}=\angle \hat{\mathbf{y}}(n)^{H} \mathbf{y}(n)$.

Using Eq. (14), the gain error is estimated as;

$\hat{d}_{n}=0.5\left(\frac{e^{j \hat{\phi}} \mathbf{y}(n)^{H} \hat{\mathbf{y}}(n)+e^{-j \hat{\phi}} \hat{\mathbf{y}}(n)^{H} \mathbf{y}(n)}{\hat{\mathbf{y}}(n)^{H} \hat{\mathbf{y}}(n)}\right)$.

Using the estimates in (14) and (15) the calibration is performed as:

$\mathbf{y}_{c}(n)=e^{-j \hat{\phi}_{n}} \mathbf{y}(n) / \hat{d}_{n}$, where $\mathbf{y}_{c}$ is the calibrated measurement. That is, a part of our optimization problem has a closed form solution and is used as such. $\square$

In following sections the proposed PC-OMP (Perturbed Calibration Orthogonal Matching Pursuit) technique is applied to two different problems. In Section 4, the proposed PC-OMP technique without any modification is applied to the frequency estimation and joint blind calibration, and simulations studies for this case are also given in the same section. In Section 5, a variant of the PCOMP algorithm Perturbed Calibration Orthogonal Matching Pursuit Direction Finding (PC-OMP-DF) is introduced for the off-grid direction finding and joint blind calibration problem, the simulation studies for this case are also given in Section 5.

\section{Application to frequency estimation}

In this section, simulation studies are performed in order to analyze the performance of the proposed method PC-OMP for the frequency estimation problem. In this case, $\mathbf{x}_{i}$ contains the frequency domain components of the corresponding measurement $\mathbf{y}_{i}$. A becomes the inverse DFT matrix with a proper size. Without loss of generality $\Psi$ is chosen to be a complex random Gaussian matrix whose elements are drawn from $N(0,1)$, although any other proper $\Psi$ matrix can be used with the proposed PC-OMP technique.

In addition to the proposed method PC-OMP, a state of the art technique, Scalable Complete Calibration technique (CCAL) [9] is used in simulations for comparison. Signal reconstruction and blind calibration performance is dependent on various parameters such as sensor number, measurement number, Signal to Noise Ratio (SNR), sparsity level and intensity of the calibration errors. For the varying values of these parameters, different scenarios and measurement sets are generated synthetically, and Monte-Carlo runs are performed. Results are analyzed and comments are drawn.

In the simulations, the proposed off-grid blind sensor calibration algorithm PC-OMP given in Table 1, the proposed algorithm without perturbation capability (Calibration Orthogonal Matching Pursuit, C-OMP) and the Scalable Complete Calibration algorithm (CCAL) [9] are used for comparison. In the simulations, Matlab ${ }^{\mathrm{TM}}$ codes for the CCAL that are available at the website given in [9] are used.

In the simulations, 20 Monte-Carlo runs are performed for each case of the scenarios. Calibrated signal reconstruction as well as the phase and the gain estimation performances are analyzed. It is important to note that the true sparse signals contains values that can be anywhere on a continuous parameter space rather than a discretized grid. While compared techniques reconstruct the signal on a discrete grid, the output of the proposed algorithm is calibrated complex amplitudes and frequency estimates of off-grid signals. Hence classical mean-square-error (MSE) metric is not a proper way of evaluating the performance of any algorithm under off-grid target scenarios. Instead, Earth mover distance (EMD) [27, 28], which is also used to measure sparse off-grid reconstructions in various applications [29,18], is used in quantitative analysis of signal reconstructions. In addition, MSE for the phase and the gain estimations are also calculated. The EMD metric is defined as follows:

- Earth Movers Distance (EMD): $P=\left(p_{i}, u_{i}\right)_{i=1}^{m}$ and $Q=$ $\left(q_{j}, v_{j}\right)_{j=1}^{n}$ are the reconstructed and the original signals respectively. $p_{i}$ and $q_{j}$ denote amplitudes, $u_{i}$ and $v_{j}$ denote values of the $i$ th and $j$ th frequencies respectively. The EMD value is the minimum work that is required for mass transportation needed for the reconstructed signal to match the original signal. The EMD is defined as [27,28]; 


$$
\operatorname{EMD}(P, Q)=\min _{F=\left\{\hat{f}_{i, j}\right\}} \frac{\sum_{i, j} \hat{f}_{i, j} d_{i, j}}{\sum_{i, j} \hat{f}_{i, j}}
$$

with constraints $\sum_{j} \hat{f}_{i, j} \leq p_{i}, \quad \sum_{i} \hat{f}_{i, j} \leq q_{i}, \quad \sum_{i, j} \hat{f}_{i, j}=$ $\min \left(\sum_{i} p_{i}, \sum_{j} q_{j}\right)$ and $\hat{f}_{i, j} \geq 0 . d_{i, j}$ is the difference between frequencies $i$ and $j . F=\left\{\hat{f}_{i, j}\right\}$ denotes set of flows. The flow $\hat{f}_{i, j}$ denotes the mass transported from the $i$ th frequency to the $j$ th. EMD is calculated as defined in [28].

Since the $\mathbf{y}=d \hat{\mathbf{y}}$ can have infinitely many solutions, the gain error estimates can be ambiguous especially for low SNR values. When this issue is not addressed, the calculated metric will be misleading. To overcome this problem, the real and the estimated signal values are normalized by its $l_{2}$ norm before EMD calculation. For the same reason, the sum of the gain error estimates are equalized to the real gain error sum before MSE calculation.

For the frequency estimation, synthetic data time domain signals with off-grid frequency components are generated, then, phase and gain errors are injected to the data as explained later in detail. Simulations are performed using this data.

\subsection{Comparative study of various SNR levels}

For comparative analysis, simulations are performed with ongrid and off-grid signals at different SNR levels. For each SNR level, synthetic data for the on-grid and the off-grid signals are generated and average results of the Monte-Carlo runs are plotted. It must be noted that CCAL is implemented using the alternating direction method of multipliers (ADMM) [9], and its performance is dependent to the proper choice of the ADMM parameter $\mu$. Although the default value is set to $\mu=50$ in the codes supplemented in [9], in the reported simulations the best performance of CCAL is obtained for $\mu=0.01$. Monte-Carlo runs are performed and results are plotted for PC-OMP, C-OMP and CCAL for $\mu=0.01$ and $\mu=50$ for comparisons. The scenario parameters are set as: the number of sensors $N_{y}=50$, the number of measurements $M=50$, the grid size (i.e. the length of $\mathbf{x}_{i}$ ) $N_{x}=50$ and the sparsity level $K=3$. Each simulated signal has $K$ frequency components. The gain errors are drawn from $N(1,0.05)$, and the phase errors are drawn from a uniform distribution between $[0,0.02 \pi]$ for each sensor. As a benchmark EMD metric is also calculated for the least squares estimator (LSE) for the signal reconstruction without performing any phase or gain error calibration.

For the on-grid signal case, the results of the compared techniques are given in Fig. 1. It can be seen that C-OMP has higher performance. CCAL gives better results for higher SNR levels, although CCAL with $\mu=50$ is very close to the LSE values. PC-OMP has a performance between CCAL and C-OMP. The C-OMP has advantage of using the exact knowledge of the grid points resulting better performance than PC-OMP which would not be applicable to off-grid signals.

For the off-grid signal case, the results of the compared techniques are given in Fig. 2. CCAL with $\mu=50$ being slightly better than LSE, the performances of CCAL and C-OMP degrades compared to the on grid case especially for the EMD metric. Meanwhile, PC-OMP has similar performance compared to the on grid case. This results shows the effectiveness of the proposed PC-OMP algorithm for the off-grid signals. Since C-OMP considers only ongrid parameter solution, its performance decreases for off-grid signals. On the other hand, PC-OMP uses parameter perturbations to estimate the off-grid signal parameters which results in better performance than C-OMP. Note that, the performance of the proposed PC-OMP decreases below $S N R=-10 \mathrm{~dB}$ which can be considered as a performance bound.
In terms of the estimation of the phase and the gain errors, PC-OMP has better results than C-OMP, while both techniques have higher performance compared to CCAL.

Runtime of the compared techniques are also calculated for computational complexity analysis. Simulation studies are performed on a PC with Intel ${ }^{\circledR}$ Core $^{\mathrm{TM}}$ i5-6200u processor and 12GB of RAM with Matlab ${ }^{\circledR}$ 2016b. Although the runtime of an algorithm depends on the implementation, they are calculated on the average as for C-OMP 0.028, PC-OMP 8.462 and CCAL 15.739 in seconds. Note that implementations of C-OMP and PC-OMP follows very closely to Table 1 and Table 2. Main algorithmic difference between C-OMP and PC-OMP is that PC-OMP uses perturbations to solve off-grid signal parameters. Runtime of C-OMP is negligible compared to PC-OMP, thus it can be said that almost all computational complexity of PC-OMP originates from perturbation process. Note that, computational complexity of the proposed PC-OMP method mainly depends on the sparsity of the signal. When the sparsity level increases, the runtime of PC-OMP decreases. To clarify the performance of the methods, algorithms are implemented in a straightforward manner, thus other factors (such as calibration errors) do not effect the computational complexity.

\subsection{Phase calibration error analysis}

Another simulation study is performed for the phase calibration error performance analysis. The same scenario parameters used as the SNR analysis with $S N R=30 \mathrm{~dB}$, a relatively high SNR value is selected to focus on the phase calibration. The phase calibration error is drawn from a uniform distribution between $[0, \phi]$ where $\phi$ is the corresponding scenario parameter.

Results are provided in Fig. 3. PC-OMP and C-OMP performance degrades as phase calibration error increases while CCAL results have small fluctuations around a mean. CCAL $\mu=0.01$ has better results than CCAL $\mu=50$. Proposed PC-OMP has the highest performance amongst compared techniques.

Note that CCAL method includes a parameter $\mu$ considerably affecting the performance with no particular method of selecting it.

Since its performance decreases after $0.3 \pi$, the phase calibration error below this level can be considered as save operating regime for the proposed PC-OMP technique.

\subsection{Gain calibration error analysis}

Gain error performances of the compared techniques are also analyzed in the simulation studies. The same scenario parameters used as the SNR analysis with $S N R=30 \mathrm{~dB}$. Monte-Carlo runs depending on gain error standard deviation are performed for two different cases with normal and log-normal distributed parameters. The standard deviation of the gain error is varied, then the scenarios are generated and results are calculated.

The results are given in Fig. 4(a) and (b). For Fig. 4(a). The gain errors are drawn from $N\left(10, d_{S T D}\right)$ where $d_{S T D}$ is the gain error standard deviation scenario parameter. Although, PC-OMP gives better results amongst the compared techniques, results of the compared techniques do not change considerably depending on the standard deviation with normal distribution. Thus, to emphasize the robustness of the proposed PC-OMP technique, another case of simulations are performed with log-normally distributed gain calibration error with mean 1 and standard deviation being scenario parameter. The results are given in Fig. 4(b), although PCOMP technique performs better than compared techniques its performance degrades for gain error standard deviation values larger than 0.5 . 


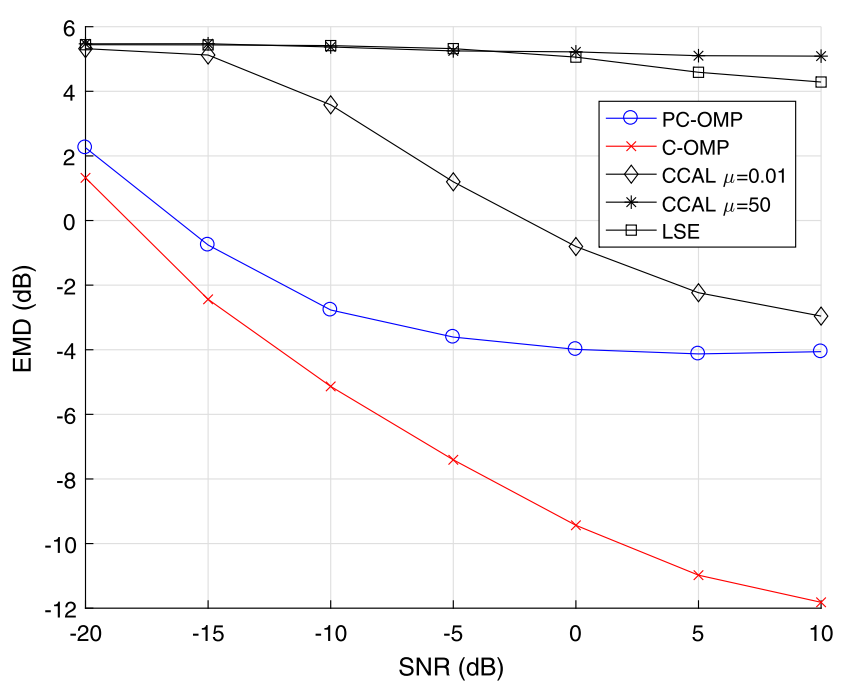

(a) EMD

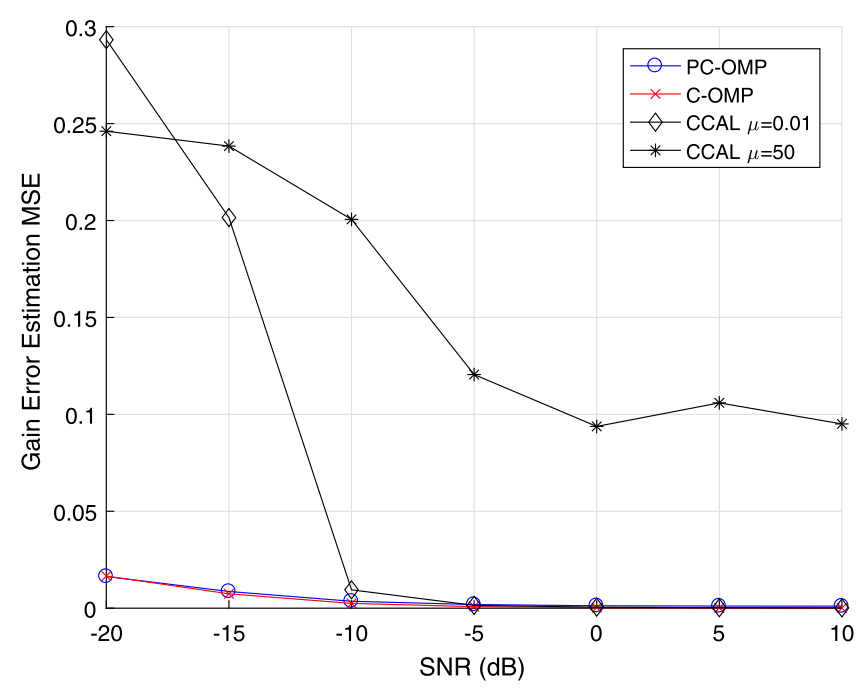

(b) Gain Error Est. MSE

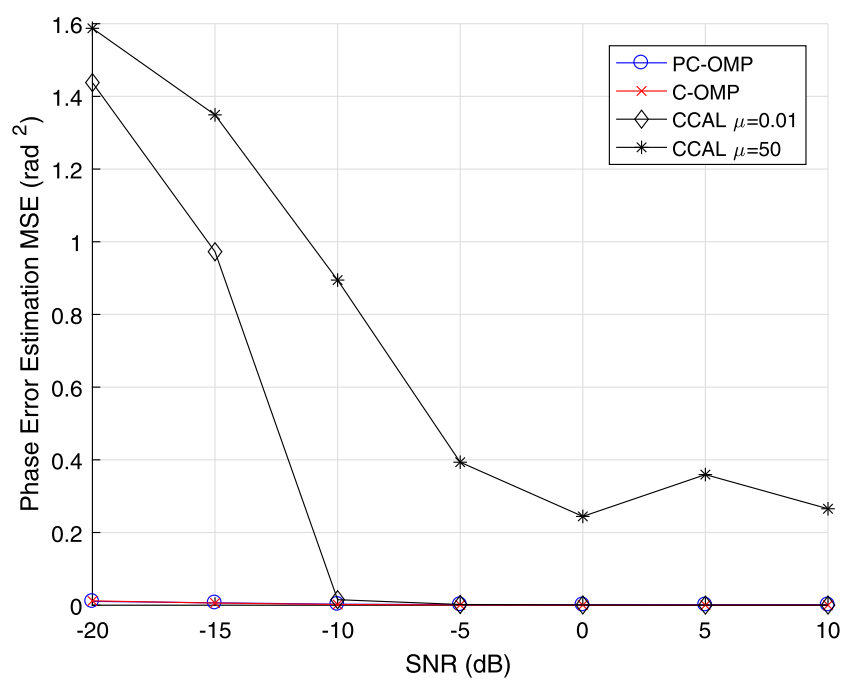

(c) Phase Error Est. MSE (Radian $\left.{ }^{2}\right)$

Fig. 1. Monte-Carlo results for the on grid case with varying SNR $=[-20,10]$ (dB). (a) EMD, (b) Gain Error Estimation MSE, (c) Phase Error Estimation MSE.

\subsection{Effects of number of measurements}

Simulation scenarios are performed to analyze the effects of the number of the measurements $M$ (i.e. the number of the snapshots) and the number of the sensors $N_{y}$. The same scenario parameters used as the SNR analysis with $S N R=30 \mathrm{~dB}$. Scenarios are generated with varying measurement and sensor numbers. The results are calculated for compared techniques and given in Fig. 5.

The results for different measurement numbers are given in Fig. 5(a). Performances of the compared techniques increases for higher measurement number levels and becomes convergent and nearly constant after $M=20$. The results for the varying sensor number are given in Fig. 5(b). It must be noted that for $N_{y}=10$, $N_{y} / N_{x}=0.2$ and for $N_{y}=50, N_{y} / N_{x}=1$. Results of the compared techniques also becomes better for the higher values of the sensor number. For both cases, PC-OMP technique yields improved results.

\subsection{Resolution performance analysis}

Resolution performance of the compared techniques are also analyzed. The same scenario parameters used as the SNR anal- ysis with $S N R=30 \mathrm{~dB}$. Two signal sources having similar absolute magnitudes with varying frequency difference levels are generated. The results are given in Fig. 6. PC-OMP performs better amongst compared techniques. Although PC-OMP performance increases slightly after the frequency difference level of 0.2 , it increases notably after level of 1 which is the initial grid cell size. It is important to emphasize that this results indicates the resolution performance of the proposed PC-OMP technique, it means that initial grid cell size effects the PC-OMP results.

\subsection{Sparsity level analysis}

Simulation scenarios are performed under varying sparsity levels $\left(K / N_{X}\right)$ between [2\%,10\%]. The same scenario parameters used as the SNR analysis with $S N R=30 \mathrm{~dB}$ and $N_{x}=50$.

The results are given in Fig. 7. It is seen that performance of the proposed PC-OMP technique decreases slowly and converges to a limit for increasing sparsity levels, and PC-OMP gives better results compared to other techniques.

Note that, under ideal conditions, when the sparsity $(K)$ is constant, increasing $N_{x}$ will result a lower $K / N_{X}$ ratio, i.e. lower sparsity level, thus it is expected to get better performance. 


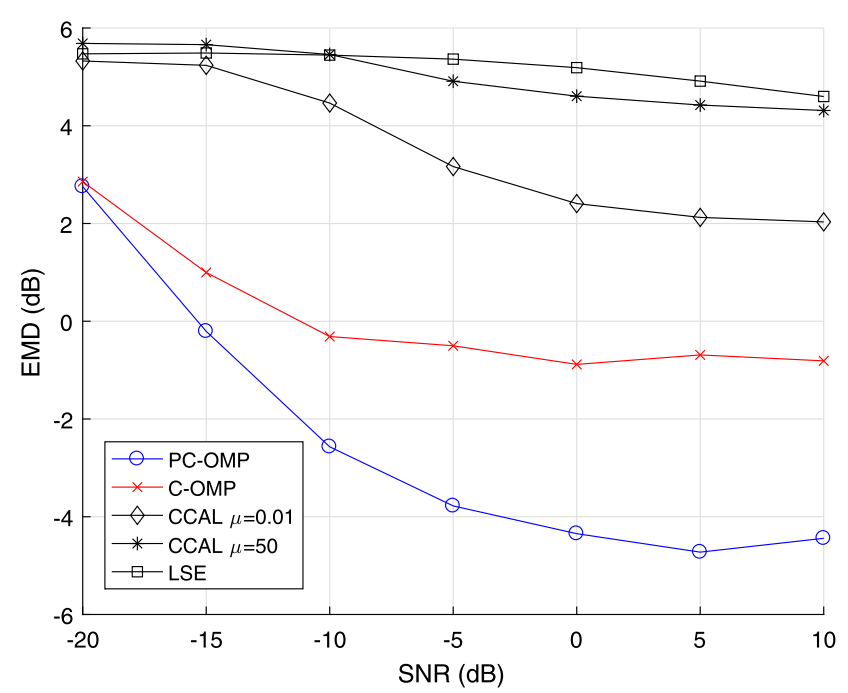

(a) EMD

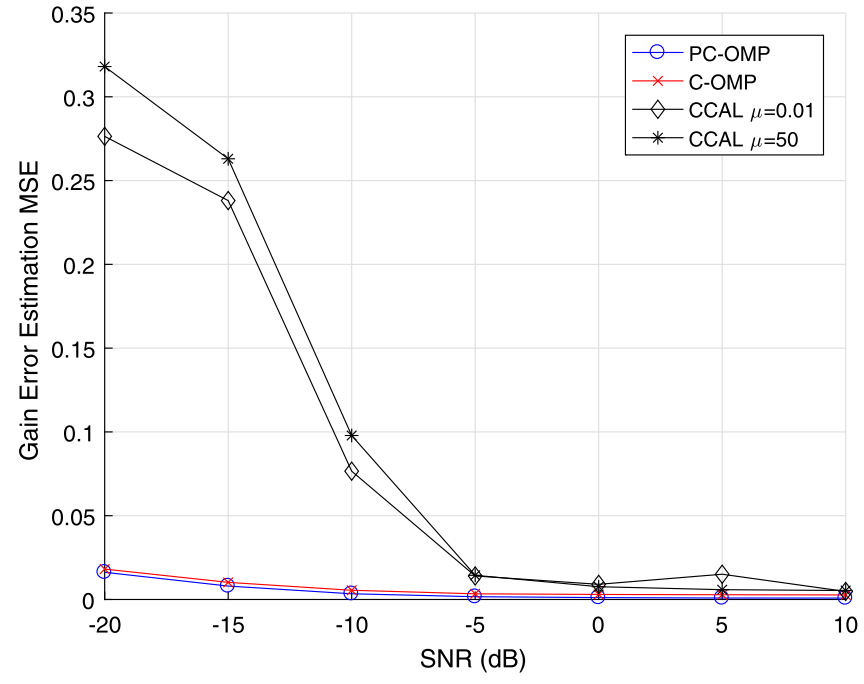

(b) Gain Error Est. MSE

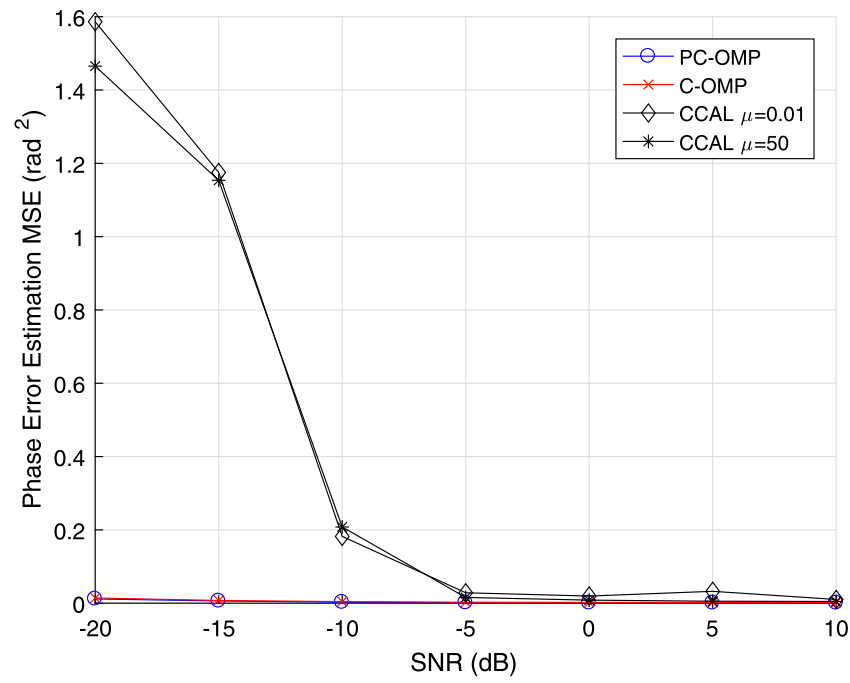

(c) Phase Error Est. MSE (Radian $\left.{ }^{2}\right)$

Fig. 2. Monte-Carlo results for the off-grid case with varying $S N R=[-20,10]$ (dB). (a) EMD, (b) Gain Error Estimation MSE, (c) Phase Error Estimation MSE.

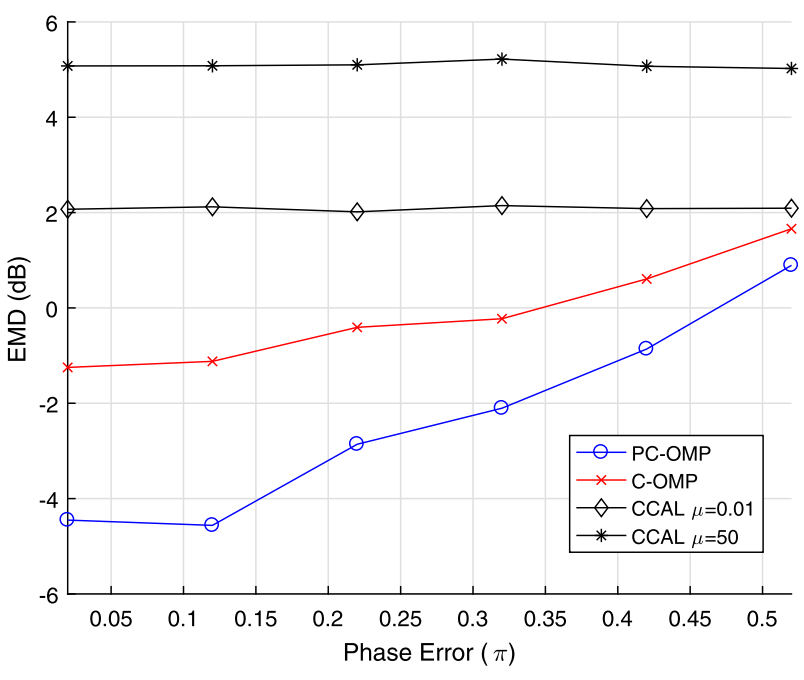

Fig. 3. EMD results for the Phase Calibration Error between $[0,0.5 \pi]$.

\subsection{Effect of the sparsity parameter}

In this section, the effect on the performance of the erroneous choice of the sparsity parameter is investigated. The same scenario parameters used as the SNR analysis with $S N R=30 \mathrm{~dB}$. PC-OMP and C-OMP are driven on Monte-Carlo runs with erroneous sparsity parameter and results are gathered. EMD for signal reconstruction and MSE for calibration error estimations are calculated and plotted. The results are given in Fig. 8. Note that the phase error estimation MSE is in radian ${ }^{2}$.

For the actual sparsity level of $K=3$, the EMD results are given in Fig. 8(a). The EMD values decreases rapidly for $K \leq 3$. For $K=1$ and $K=2$, although PC-OMP has slightly better performance, the EMD results of C-OMP and PC-OMP are very close. The difference is at the highest value for the true sparsity level input at $K=3$. For the inputs higher than $K=3$ signal reconstruction performance decreases as EMD increases. While PC-OMP and C-OMP results are close for $K \geq 4$, PC-OMP has better performance.

The calibration error performances are given in Fig. $8(\mathrm{~b})$. The phase and gain error estimation MSE decreases rapidly when $K \leq 3$ for both techniques. Note that unlike the EMD, calibration error estimation MSE decreases for $K \geq 4$. For $K \geq 4$, the OMP selects noise-generated atoms which decreases EMD values while cali- 


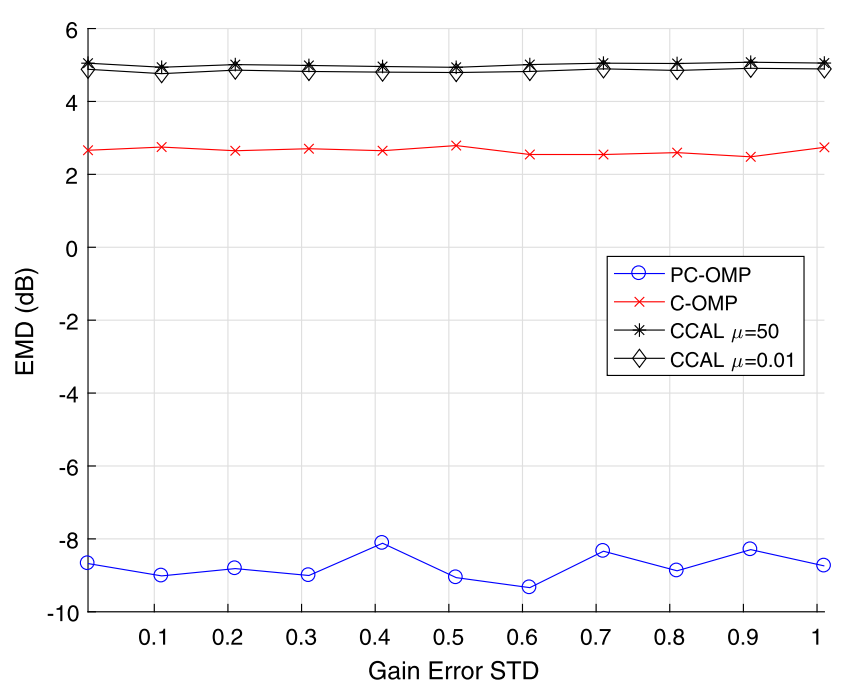

(a) Normal Distributed

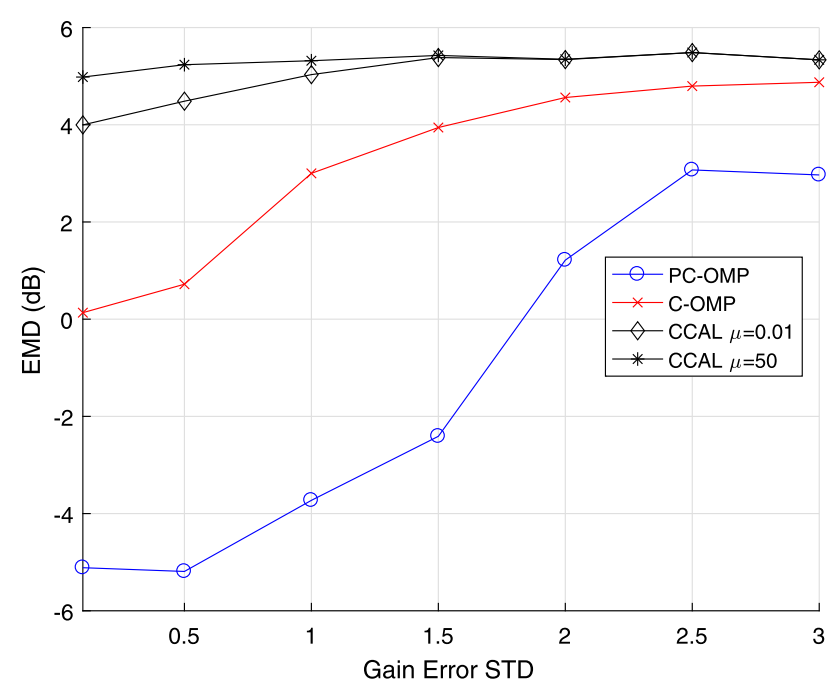

(b) Log-Normal Distributed

Fig. 4. EMD results for the Gain Calibration Error Standard Deviation for (a) Normal Distributed [0, 1], (b) Log-Normal Distributed [0.01, 3].

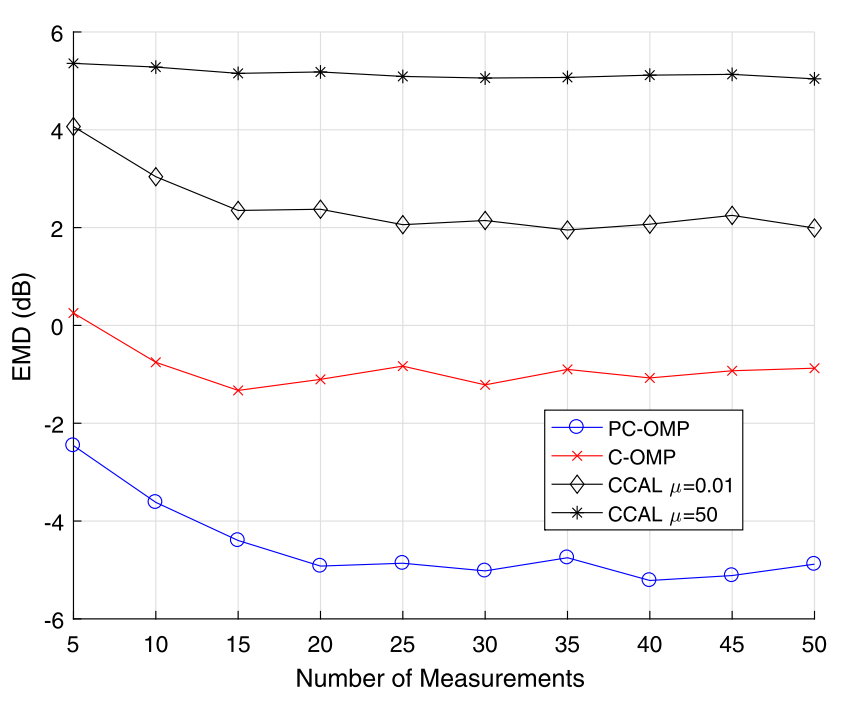

(a) Measurement number

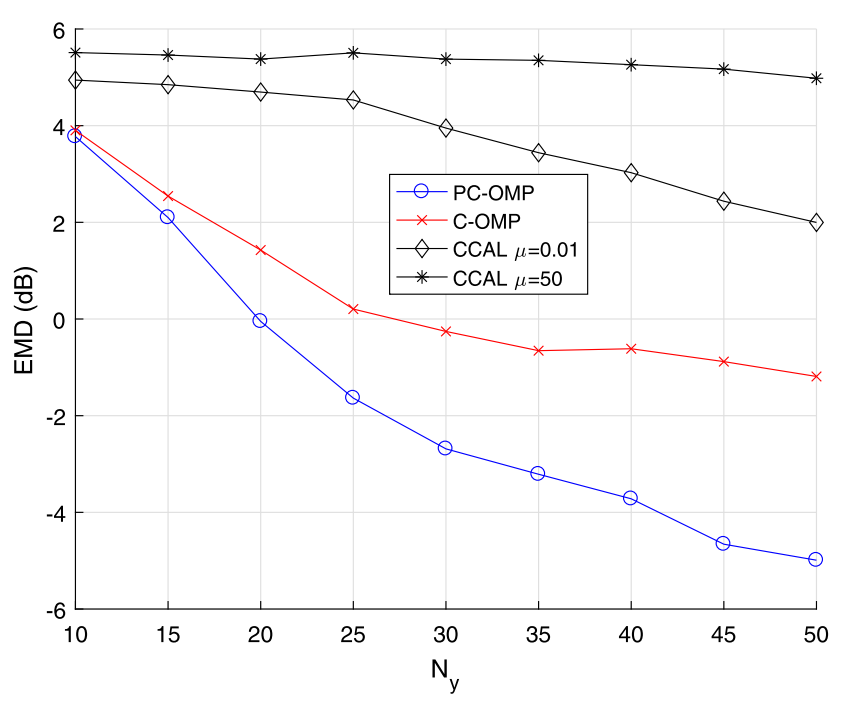

(b) Sensor number

Fig. 5. EMD results for (a) Measurement number [5, 50], (b) Sensor number $[10,50]$.

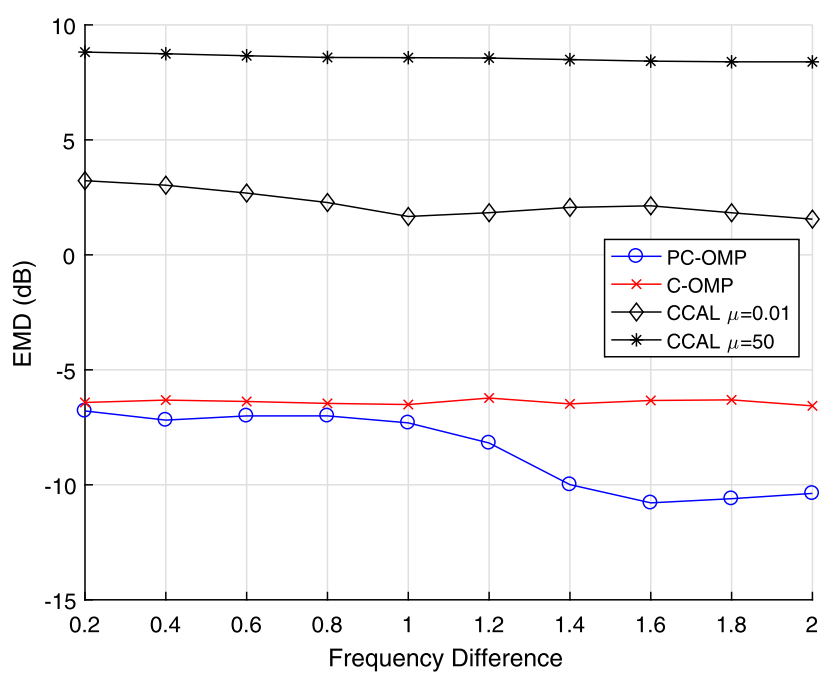

Fig. 6. EMD results for the Frequency Difference levels between $[0.2,2 \pi]$. bration performance is not affected. This shows that the signal reconstruction and therefore the blind calibration are performed robustly even with the erroneous choice of the sparsity level. In addition, PC-OMP has better results at all the erroneous sparsity levels. $\square$

In this section, the performance of the proposed technique PCOMP is demonstrated through comprehensive simulation studies, and its effectiveness is represented using different scenario cases amongst compared techniques. In the next section, the proposed technique is applied to the direction finding problem, and its performance is investigated for this case.

\section{Application to direction of arrival estimation}

There are different studies on blind sensor calibration applied to direction finding in the literature. In [30], a high resolution direction finding and blind calibration method is proposed. Iteratively, the directions are estimated first using Eigen value decomposition, then the blind calibration is performed. Mutual coupling between sensors is also estimated as well as the gain and phase errors [30]. In [31], a non-iterative algebraic solution is developed 
for the blind sensor calibration and beam forming. In this method, the sensor locations are assumed to be known, and the gain and phase calibration errors of the sensors are estimated. Similarly, the signal estimation and the blind sensor calibration are performed separately in [32]. In [33], a blind calibration method using independent component analysis is studied which considers the gain and the phase errors for a linear array. In [34], compressive sensing based array self-calibration algorithms are proposed for direction finding in which the signal parameters and unknown complex sensor gains are estimated in an alternating manner. In [35], a sparse DOA estimation approach is proposed using arrays with unknown perturbations on sensor locations. DOA and perturbation values are solved iteratively. In [36], a sparsity based iterative method is developed for the off-grid DOA estimation with co-prime arrays. In [37], a method direction of arrival estimation and gain and phase error correction is proposed based on the exploiting the knowledge on the signal eigenvectors.

High resolution and subspace methods for blind sensor calibration and direction finding do not exploit the compressive sensing instruments, while CS based array calibration methods generally do not consider the off-grid problem. To address these issues, we

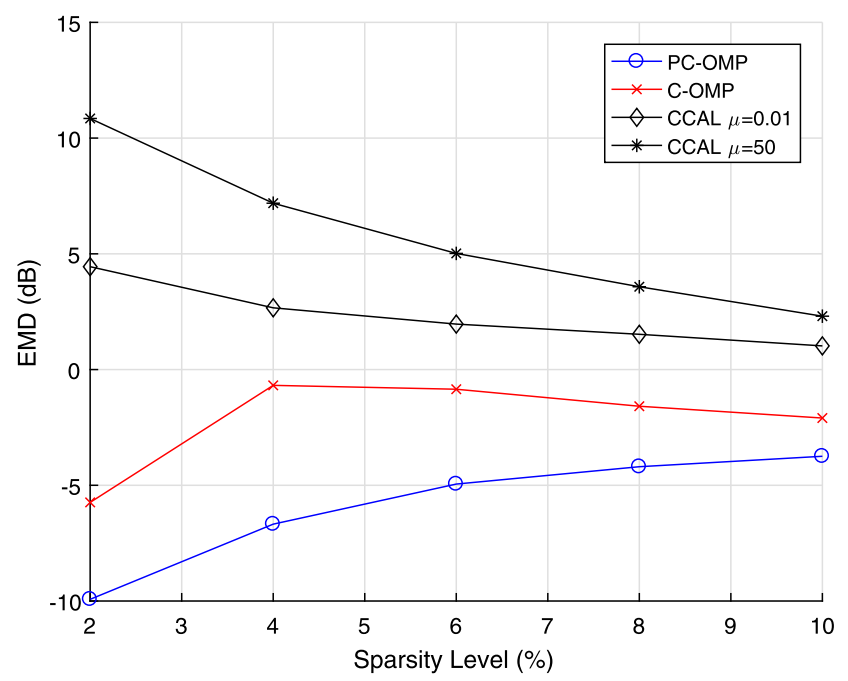

Fig. 7. EMD results for different sparsity levels between [2\%, 10\%].

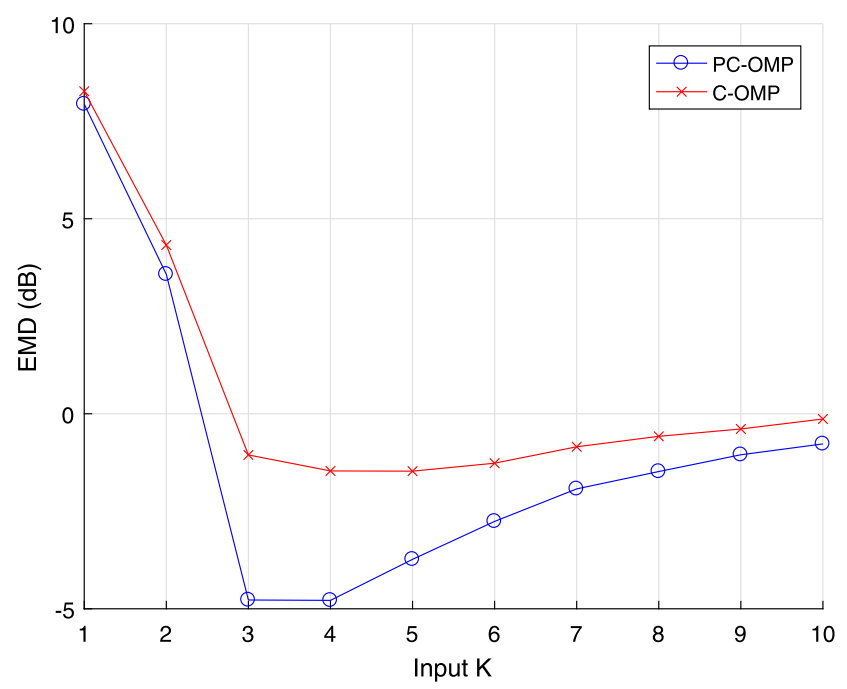

(a) EMD propose a compressive sensing based off-grid direction finding and blind calibration method.

In this section, a variant of the PC-OMP algorithm Perturbed Calibration Orthogonal Matching Pursuit Direction Finding (PC-OMP-DF) is introduced for the off-grid direction finding and joint blind calibration problem. The method is capable of estimating the off-grid direction of the signals while jointly performing blind calibration to estimate and correct the gain and the phase errors of the sensors. In the next section the algorithm of the proposed $\mathrm{PC}-\mathrm{OMP}-\mathrm{DF}$ is explained and simulation analysis is given. In the next subsection, the PC-OMP-DF technique is explained and its variations from PC-OMP is given in detail.

\subsection{Sparsity based off-grid direction finding and blind calibration}

Direction finding problem is solved using an Uniform Linear Array (ULA). Assuming the sources are sparse in direction dimension and using ULA, the equivalent measurement model matrix $\mathbf{G}$ in Eq. (2) becomes the array manifold matrix and expressed as [38]:

$\mathbf{G}=\left[\begin{array}{llll}\mathbf{a}\left(\theta_{1}\right) & \mathbf{a}\left(\theta_{2}\right) & \ldots & \mathbf{a}\left(\theta_{K}\right)\end{array}\right]$,

where $\mathbf{a}\left(\theta_{k}\right)=[1 \exp (-j a) \exp (-j 2 a) \ldots \exp (-j(N-1) a)]^{T}$ is array response at angle $\theta_{k}$, and $a=2 \pi d / \lambda \sin \left(\theta_{k}\right) . a$ and $d$ the phase difference and distance between successive elements in ULA respectively; $\lambda$ the wavelength.

The proposed PC-OMP algorithm can be applied to direction finding problem using the equivalent measurement model matrix in Eq. (18) without any other variation. On the other hand, with a sufficiently small total measurement time, it is possible to consider that the directions are constant over snapshots, since they do not change considerably. Using the constant directions assumption, in this section, the proposed PC-OMP algorithm is adopted to direction finding problem with some variations. If the directions are expressed over a grid having a certain resolution (i.e., $1^{\circ}$ ), the constant assumed directions of different snapshots will form a group over the grid.

In the proposed PC-OMP-DF technique, the atom selection in OMP is performed jointly for all exploiting the group property and using the group [39] and the block [40] sparsity ideas, the following projection technique is proposed for the atom selection in OMP;

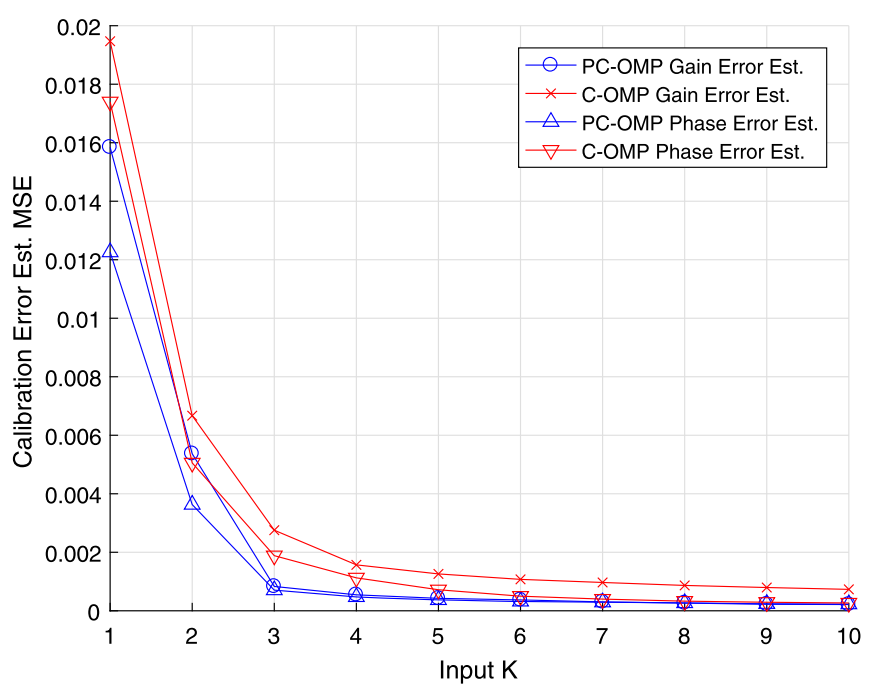

(b) Calibration Error Est. MSE

Fig. 8. Results for the erroneous sparsity parameter (a) EMD, (b) Calibration Error Est. MSE. 
$\hat{p}=\max \sum_{i=1}^{D}\left|\mathbf{G}^{H} \mathbf{y}_{i}\right|$.

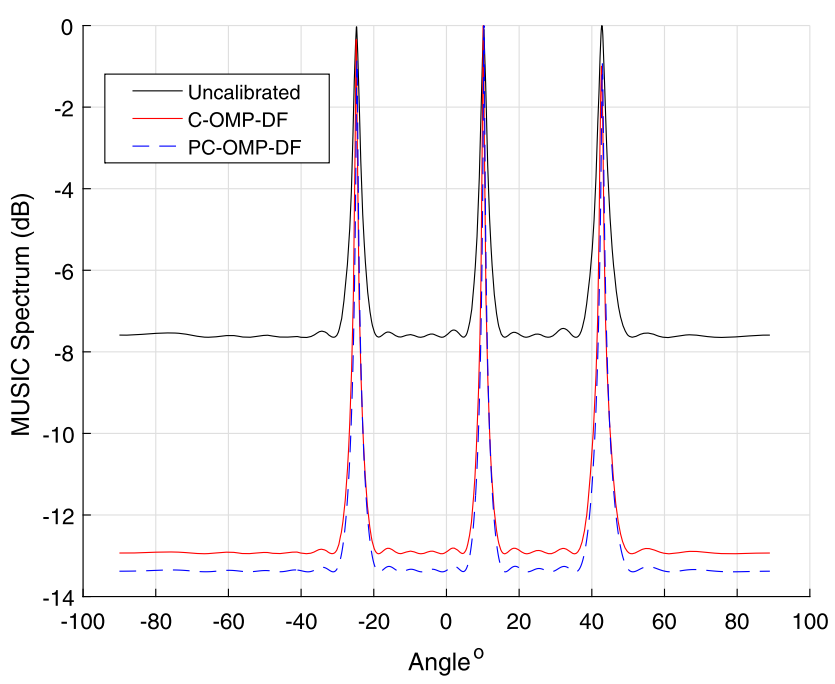

Fig. 9. Uncalibrated and calibrated MUSIC spectrums.

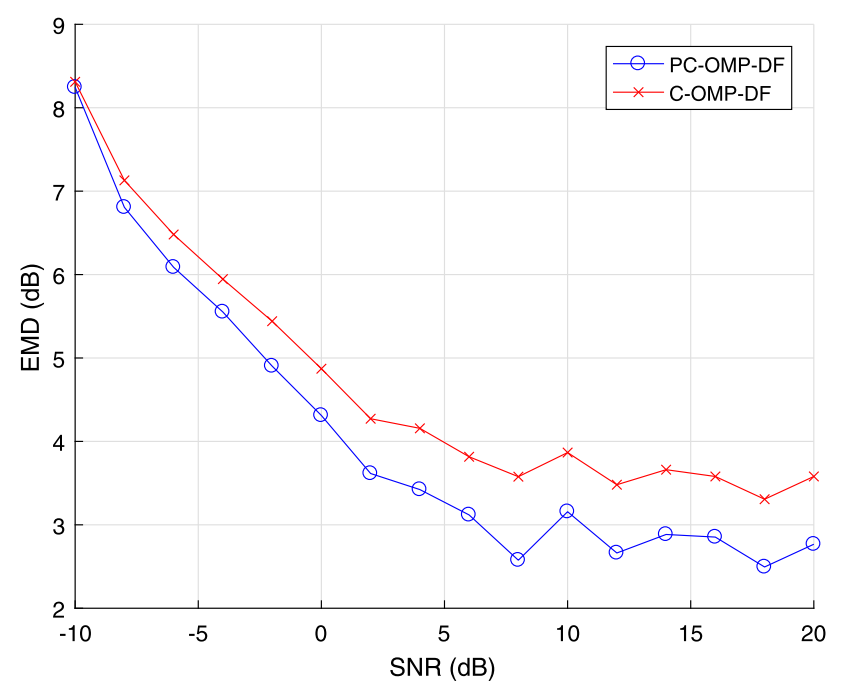

(a) EMD

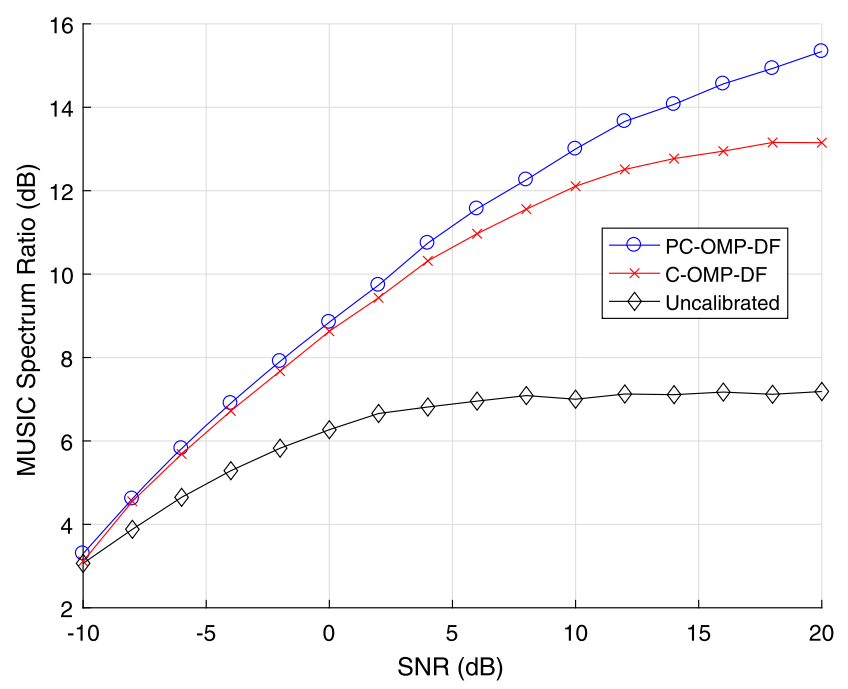

(c) Max/Min Ratio
The same atom $\hat{p}$ which is the maximum of the projection in Eq. (19) is used for every snapshot. The perturbation process is performed similar to PC-OMP with small variations. After each perturbation, angular average of the perturbed directions are calculated yielding single direction results for each atom selected using Eq. (19). The resulting directions are registered to corresponding snapshots. This procedure is repeated until a stopping criterion is met. The blind calibration is applied similar to PC-OMP.

\subsection{Simulations}

Simulations are performed to investigate the performance of the proposed PC-OMP-DF technique. A variant of PC-OMP-DF without perturbation capability, Calibration OMP-DF method (C-OMPDF) is used in simulations to demonstrate the effectiveness of the perturbation more clearly.

Synthetic data is generated using a uniform linear array (ULA). Array beam pattern, resolution and accuracy is dependent on the array size and the direction of the signals [38]. To reduce these effects, a constant number of signals $K=3$ is used with stationary directions of $-25^{\circ}, 9.57^{\circ}$ and $42.48^{\circ}$ which are randomly selected with no significant effect on performance.

In simulations, several metrics are used to evaluate the performance of the proposed techniques. The Earth Movers Distance

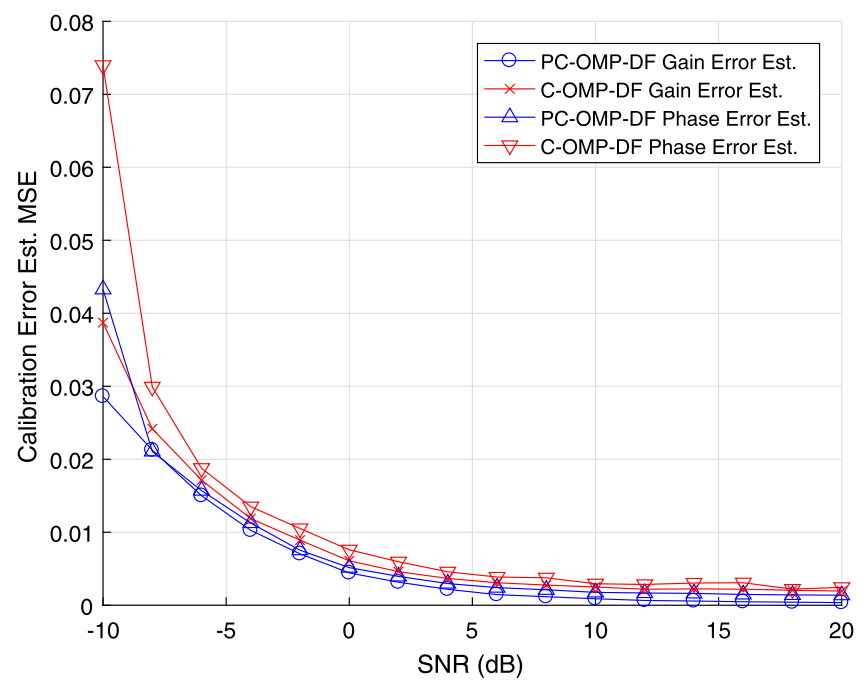

(b) Calibration Error Est. MSE

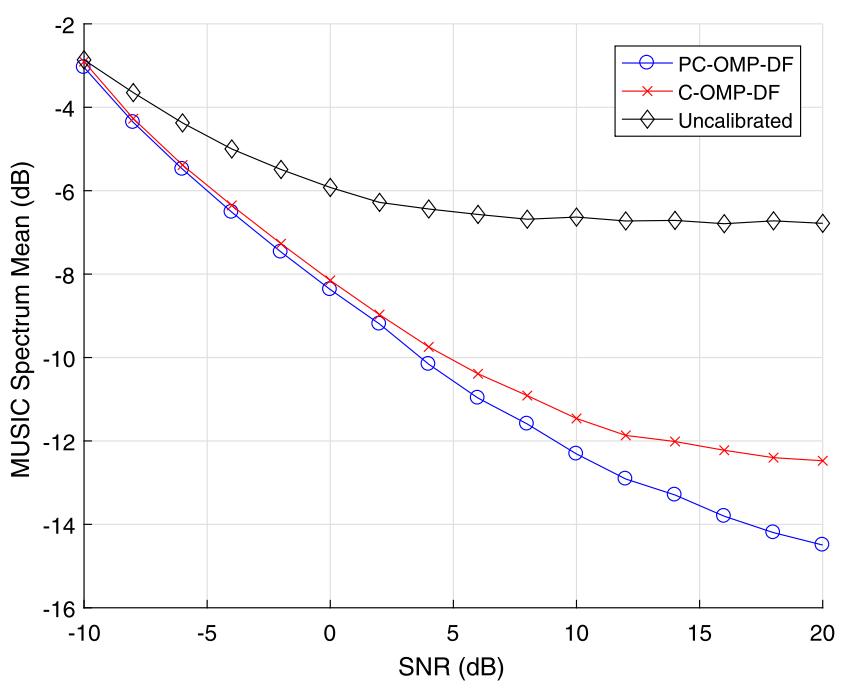

(d) Spectrum Mean

Fig. 10. Results for varying SNR (a) EMD, (b) Calibration Error Est. MSE, (c) MUSIC Spectrum Max/Min Ratio, (d) MUSIC Spectrum Mean. 


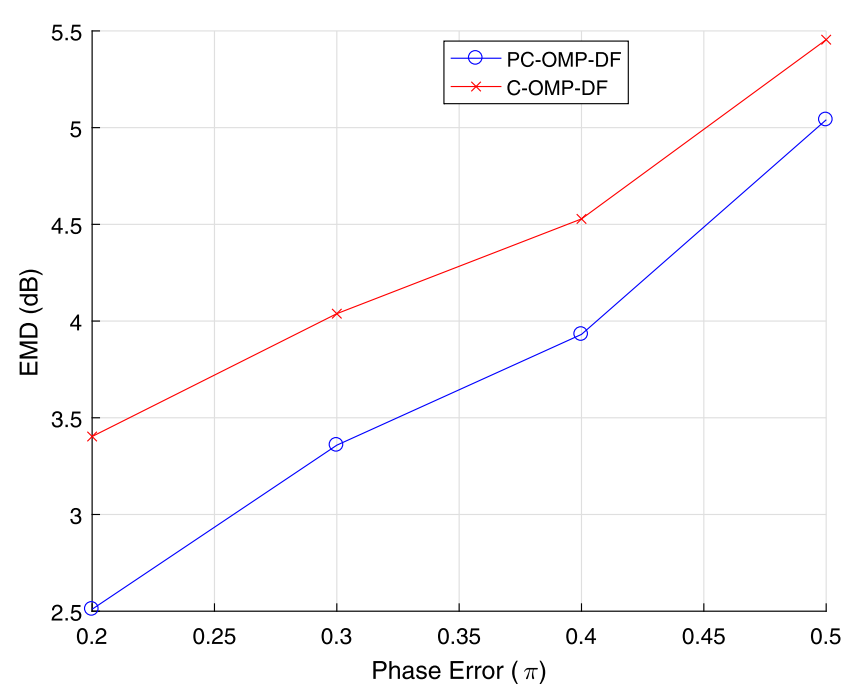

(a) EMD

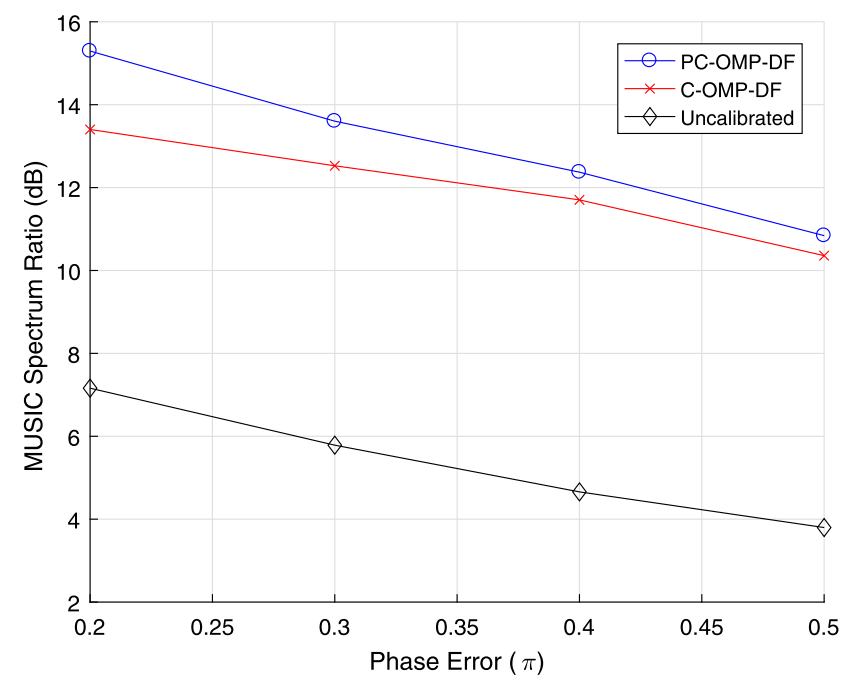

(c) Max/Min Ratio

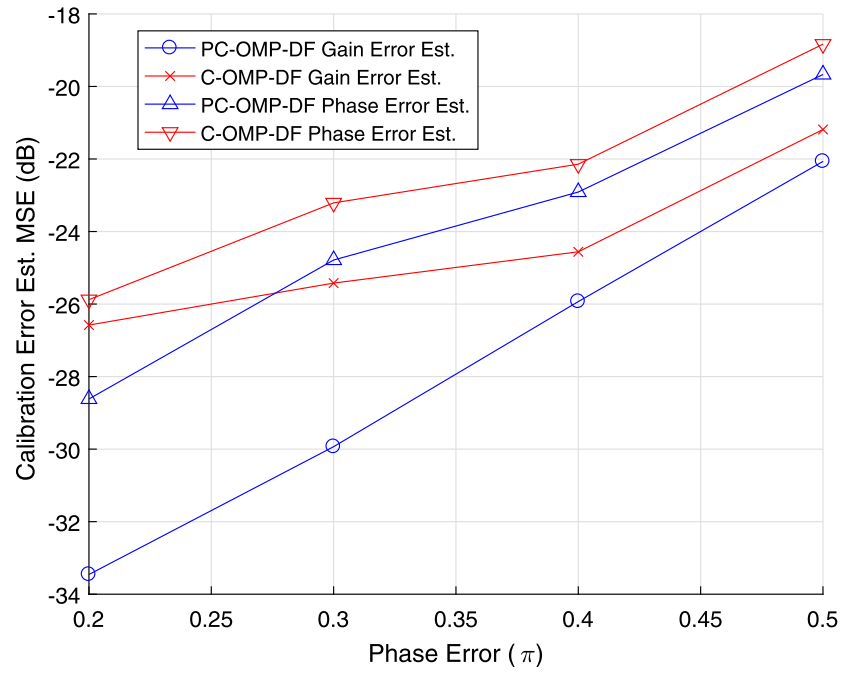

(b) Calibration Error Est. MSE (dB)

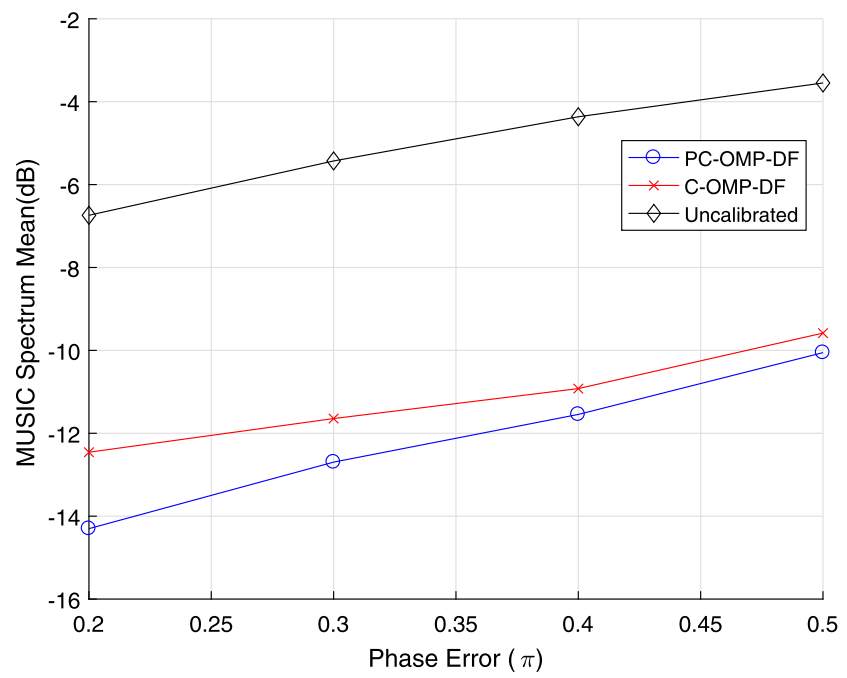

(d) Spectrum Mean

Fig. 11. Results for varying SNR (a) EMD, (b) Calibration Error Est. MSE (dB), (c) MUSIC Spectrum Max/Min Ratio, (d) MUSIC Spectrum Mean.

(EMD) between true and estimated directions are calculated. The MSE of the gain and the phase errors are also calculated.

In addition to mentioned metrics, the MUSIC spectrum of the measured and calibrated signals can also be exploited as a performance indicator. A sample MUSIC spectrum of true and calibrated signals is given in Fig. 9 with $S N R=20 \mathrm{~dB}$ and an ULA with 40 elements. It is seen that calibrated spectrums are much more sharper than the uncalibrated spectrum. The calibration increases the coherent signal processing gain which results in sharper spectrum. Using this result, the following indicators are also calculated for the uncalibrated and the calibrated spectrums;

- The mean value of the spectrum,

- The ratio of the maximum and minimum values of the spectrum.

\subsubsection{SNR analysis}

For the DF performance analysis, first case of simulations are performed for varying SNR levels. For each scenario parameter, 50 Monte-Carlo runs are evaluated and results are plotted. Sensor and snapshot numbers are chosen as 40 , number of the sources is $K=3$. The gain calibration error of the sensors is in- dependently drawn from $N(1,0.1)$, and the phase calibration error is drawn independently from a uniform distribution between $[0,0.2 \pi]$.

EMD direction finding (DF) results for varying SNR are given in Fig. 10(a). The performances of the compared techniques improves for higher SNR values. It is seen that PC-OMP-DF technique has better performance compared to C-OMP-DF. This result demonstrates the effectiveness of the perturbation procedure. The gain and the phase calibration error estimation performance increases for higher SNR levels. For the calibration error estimation, PC-OMPDF also yields improved results.

The results calculated using the uncalibrated and the calibrated signals' MUSIC spectrums are given in Fig. 10(c) and (d) respectively. The maximum and minimum value ratio of the MUSIC spectrums are given in Fig. 10(c). Higher value of this ratio indicates better calibration. It is seen that the ratios with calibrated spectrums using PC-OMP-DF and C-OMP-DF are higher than those of the uncalibrated spectrum. This result shows the improvement of the calibration by proposed techniques. Similarly, the mean values of the uncalibrated and the calibrated spectrums are given in Fig. 10(d). Smaller value indicates better calibration for this parameter. It is seen that calibration results in better performance. 


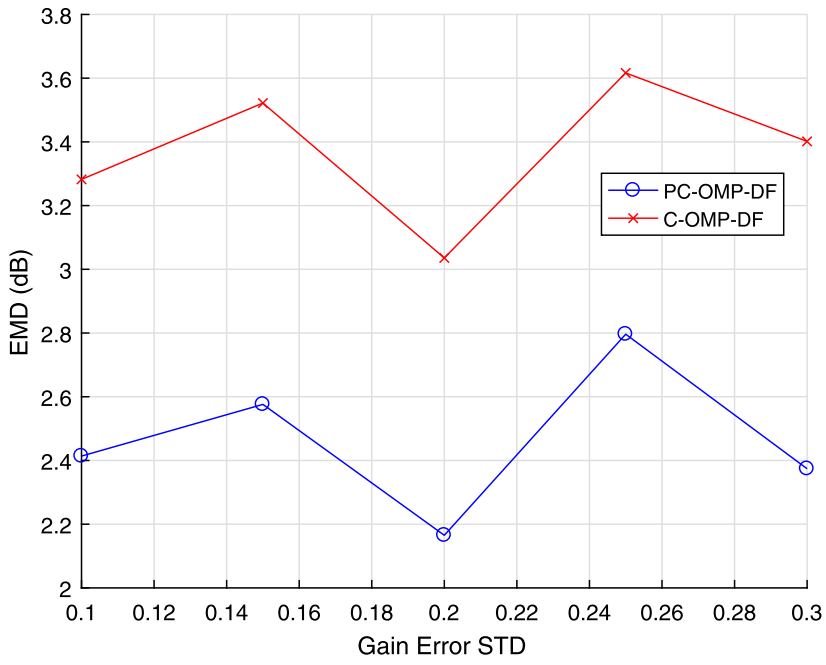

Fig. 12. EMD results for gain error standard deviation $[0.1,0.3]$.

Both parameters have better results compared to the uncalibrated case for increasing SNR for both in Fig. 10(c) and (d). For all of the cases, PC-OMP-DF has higher performance than C-OMP-DF algorithm, and the performance difference increases for higher SNR. This is also another proof for the effectiveness of the proposed PCOMP-DF method.

Runtime of the compared techniques are also calculated for computational complexity analysis. Simulation studies are performed on a PC with Intel ${ }^{\circledR}$ Core $^{\mathrm{TM}}$ i5-6200u processor and 12 GB of RAM with Matlab ${ }^{\circledR}$ 2016b. In seconds, runtime of C-OMP-DF is calculated as 0.028 , and for PC-OMP-DF 2.375. Since runtime of C-OMP-DF is negligible, it can be said that almost all computational complexity of PC-OMP-DF originates from perturbation process. Similar to PC-OMP, computational complexity of the proposed PC-OMP-DF method mainly depends on the sparsity of the signal.

\subsubsection{Phase calibration error analysis}

In this section, simulation results for the varying level of phase calibration error are given. Scenario parameters are the same as in Section 5.2.1 with $S N R=20(\mathrm{~dB})$. Phase calibration error is drawn independently from a uniform distribution between 0 and corresponding scenario parameter.

The results for varying phase calibration error are given in Fig. 11. It is seen that for the phase calibration errors larger than $0.4 \pi$, the performances of the compared techniques degrades considerably. Although results are very close, PC-OMP-DF gives better performance under the phase calibration error level of $0.4 \pi$.

\subsubsection{Gain calibration error analysis}

In this section, simulation results for the varying level of gain calibration error standard deviation are given. Scenario parameters are the same as in Section 5.2.1 with $S N R=20(\mathrm{~dB})$. The gain errors are drawn from $N\left(1, d_{S T D}\right)$ where $d_{S T D}$ is the gain error standard deviation scenario parameter.

Results are given in Fig. 12. Although the performances of the compared techniques does not change considerably for different SNR levels, it can be seen that PC-OMP-DF gives better results.

\section{Conclusions}

Compressed Sensing (CS) based techniques represent the signal on a discretized grid which results in, due to continuous na- ture of the signals, the off-grid problem, which significantly affects the performance. Improper calibration is also another issue which can cause performance degradation. In this work, a sparsity based off-grid blind calibration method is proposed (Perturbed Calibration Orthogonal Matching Pursuit, PC-OMP) and with some variations it is applied to frequency estimation and direction finding problems. The proposed techniques are capable of estimating the off-grid signal parameters (such as frequency or direction) and complex gains while performing the blind calibration to estimate and calibrate the gain and the phase errors jointly. The signal estimation and blind calibration procedures are performed alternately in an iterative manner. The methods use a gradient descent approach to solve the off-grid signal parameters. The simulation results show that the proposed techniques PC-OMP and PC-OMP-DF provide significant performance improvements.

\section{References}

[1] D. Donoho, Compressed sensing, IEEE Trans. Inf. Theory 52 (4) (2006) $1289-1306$.

[2] E. Candes, J. Romberg, T. Tao, Robust uncertainty principles: exact signal reconstruction from highly incomplete frequency information, IEEE Trans. Inf. Theory 52 (2006) 489-509.

[3] R. Baraniuk, M. Davenport, R. DeVore, M. Wakin, A simple proof of the restricted isometry property for random matrices, Constr. Approx. 28 (3) (2008) 253-263.

[4] M. Cetin, I. Stojanovic, N. Onhon, K. Varshney, Sparsity-driven synthetic aperture radar imaging: reconstruction, autofocusing, moving targets, and compressed sensing, IEEE Signal Process. Mag. 31 (4) (2014) 27-40.

[5] J. Ender, A brief review of compressive sensing applied to radar, in: 2013 14th International Radar Symposium, vol. 1, 2013, pp. 3-16.

[6] L. Balzano, R. Nowak, Blind calibration of sensor networks, in: 6th International Symposium on Information Processing in Sensor Networks, April 2007.

[7] B.C. Ng, C.M.S. See, Sensor-array calibration using a maximum likelihood approach, IEEE Trans. Antennas Propag. 44 (1996) 827-835.

[8] R. Mignot, L. Daudet, F. Ollivier, Room reverberation reconstruction interpolation of the early part using compressed sensing, IEEE Trans. Audio Speech Lang. Process. 21 (11) (2013) 2301-2312.

[9] C. Bilen, G. Puy, R. Gribonval, L. Daudet, Convex optimization approaches for blind sensor calibration using sparsity, IEEE Trans. Signal Process. 62 (2014) 4847-4856.

[10] H. Shen, M. Kleinsteuber, C. Bilen, R. Gribonval, A conjugate gradient algorithm for blind sensor calibration in sparse recovery, in: IEEE International Workshop on Machine Learning for Signal Processing, September 2013.

[11] J. Lipor, L. Balzano, Robust blind calibration via total least squares, in: IEEE International Conference on Acoustic, Speech and Signal Processing (ICASSP), 2014.

[12] S. Wijholds, S. Chiarucci, Blind calibration of phased arrays using sparsity constraints on the signal model, in: European Signal Processing Conference (EUSIPCO), 2016

[13] C. Wang, P. Ramanathan, K.K. Saluja, Moments based blind calibration in mobile sensor networks, in: 2008 IEEE International Conference on Communications, 2008, pp. 896-900.

[14] V. Cambareri, L. Jacques, A non-convex blind calibration method for randomised sensing strategies, in: 2016 4th International Workshop on Compressed Sensing Theory and Its Applications to Radar, Sonar and Remote Sensing (CoSeRa), 2016, pp. 16-20.

[15] V. Cambareri, A. Moshtaghpour, L. Jacques, A greedy blind calibration method for compressed sensing with unknown sensor gains, in: 2017 IEEE International Symposium on Information Theory (ISIT), 2017, pp. 1132-1136.

[16] Y. Chi, L. Scharf, A. Pezeshki, R. Calderbank, The sensitivity to basis mismatch of compressed sensing in spectrum analysis and beamforming, in: Sixth Workshop on Defense Applications of Signal Processing (DASP), Lihue, HI, Oct. 2009.

[17] O. Teke, A. Gurbuz, O. Arikan, A robust compressive sensing based technique for reconstruction or sparse radar scenes, Digit. Signal Process. 27 (2013) 23-32.

[18] S. Camlica, A.C. Gurbuz, O. Arikan, Autofocused spotlight sar image reconstruction of off-grid sparse scenes, IEEE Trans. Aerosp. Electron. Syst. 53 (2017) 1880-1892.

[19] H. Zhu, G. Leus, G. Giannakis, Sparsity-cognizant total least-squares for perturbed compressive sampling, IEEE Trans. Signal Process. 59 (5) (2011) 2002-2016.

[20] G. Tang, B. Bhaskar, P. Shah, B. Recht, Compressed sensing off the grid, IEEE Trans. Inf. Theory 59 (11) (2013) 7465-7490.

[21] O. Teke, A. Gurbuz, O. Arikan, Perturbed orthogonal matching pursuit, IEEE Trans. Signal Process. 61 (24) (2013) 6220-6231. 
[22] C. Ekanadham, D. Tranchina, E.P. Simoncelli, Recovery of sparse translationinvariant signals with continuous basis pursuit, IEEE Trans. Signal Process. 59 (10) (2011) 4735-4744.

[23] J. Tropp, A. Gilbert, Signal recovery from random measurements via orthogonal matching pursuit, IEEE Trans. Inf. Theory 53 (12) (Dec. 2007) 4655-4666.

[24] H.T. Wu, J.F. Yang, F.K. Chen, Source number estimators using transformed gerschgorin radii, IEEE Trans. Signal Process. 43 (6) (1995) 1325-1333.

[25] J. Liang, D. Liu, Joint estimation of source number and doa using simulated annealing algorithm, Digit. Signal Process. 20 (3) (2010) 887-899.

[26] K. Liu, H.C. So, J.P.C. da Costa, F. Römer, L. Huang, Efficient source enumeration for accurate direction-of-arrival estimation in threshold region, Digit. Signal Process. 23 (5) (2013) 1668-1677.

[27] H. Ling, K. Okada, An efficient Earth mover's distance algorithm for robust histogram comparison, IEEE Trans. Pattern Anal. Mach. Intell. 29 (5) (2007) $840-853$.

[28] Y. Rubner, C. Tomasi, L.J. Guibas, The Earth mover's distance as a metric for image retrieval, Int. J. Comput. Vis. 40 (2) (2000) 99-121.

[29] L. Schmidt, C. Hegde, P. Indyk, The constrained Earth mover distance model, with applications to compressive sensing, in: 10th Intl. Conf. on Sampling Theory and Appl., SAMPTA, 2013.

[30] A. Khallaayoun, R.J. Weber, Y. Huang, A blind iterative calibration method for high resolution DOA estimation, in: IEEE Military Communications Conference, MD, USA, January 2011

[31] B. Demissie, Algebraic method for deterministic blind beamforming with unknown receiver gain and phases, in: IEEE International Conference on Signal Processing and Communications (ICSPC 2007), Dubai, November 2007.

[32] J. Liu, X. Wu, W.J. Emery, L. Zhang, C. Li, K. Ma, Direction-of-arrival estimation and sensor array error calibration based on blind signal separation, IEEE Signal Process. Lett. 24 (1) (2017) 7-11.

[33] J. Kim, H.J. Yang, B.W. Jung, J. Chun, Blind calibration for a linear array with gain and phase error using independent component analysis, Antennas Wirel. Propag. Lett. 9 (2010) 1259-1262.

[34] B. Friedlander, T. Strohmer, Bilinear compressed sensing for array selfcalibration, in: 2014 48th Asilomar Conference on Signals, Systems and Computers, 2014, pp. 363-367.

[35] A. Koochakzadeh, P. Pal, Sparse source localization using perturbed arrays via bi-affine modeling, Digit. Signal Process. 61 (2017) 15-25, special Issue on Coprime Sampling and Arrays.

[36] F. Sun, Q. Wu, Y. Sun, G. Ding, P. Lan, An iterative approach for sparse directionof-arrival estimation in co-prime arrays with off-grid targets, Digit. Signal Process. 61 (2017) 35-42, special Issue on Coprime Sampling and Arrays.

[37] A. Liu, G. Liao, An eigenvector based method for estimating DOA and sensor gain-phase errors, Digit. Signal Process. 79 (2018) 116-124.

[38] H.V. Trees, Optimum Array Processing, John Wiley and Sons, NY, USA, 2002

[39] A. Majumdar, R. Ward, Fast group sparse classification, in: IEEE Pacific Rim Conference on Communications, Computers and Signal Processing, vol. 34, August 2009, pp. 11-16.

[40] Y. Eldar, P. Kuppinger, H. Bolcskei, Block-sparse signals uncertainty relations and efficient recovery, IEEE Trans. Signal Process. 58 (6) (2010) 3042-3054.

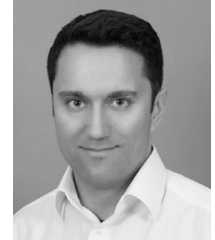

Sedat Camlica was born in 1982 Izmir, Turkey. In 2006, he received the B.Sc. degree in Telecommunications Engineering from Istanbul Technical University, Istanbul, Turkey. He received the M.Sc. degree in 2009 in Electrical and Electronics Engineering from the Middle East Technical University, Ankara, Turkey. He received the Ph.D. degree in 2017 in Electrical and Electronics Engineering at TOBB University of Economics and Technology, Ankara, Turkey. Since 2006 he is serving at ASELSAN A.S., Radar \& Electronic Warfare Systems Business Sector, Ankara, Turkey. His research interests include compressive sensing applications, array and radar signal processing, target tracking, remote sensing and imaging.

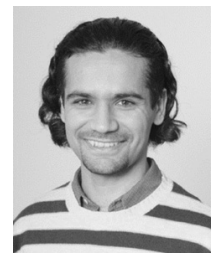

Imam Samil Yetik received the B.S. degree from Bogazici University, Istanbul, Turkey, in 1998, the M.S. degree from Bilkent University in 2000, and the Ph.D degree in electrical engineering from the University of Illinois at Chicago, Chicago, IL, USA, in 2004. Between 2005 and 2006, he was a post-doctoral at the University of Illinois-Chicago and University of California at Davis. He served as a faculty member at Illinois Institute of Technology between 2006 and 2011. Currently, he is an Associate Professor with the Department of Electrical and Electronics Engineering at TOBB ETU. His research interests are in the areas of signal and image processing with statistical approaches and machine learning techniques applied to biomedicine and defense systems.

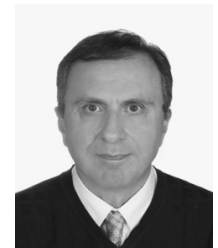

Orhan Arikan was born in 1964 in Manisa, Turkey In 1986, he received his B.Sc. degree in Electrical and Electronics Engineering from the Middle East Technical University, Ankara, Turkey. He received both his M.S. and Ph.D. degrees in Electrical and Computer Engineering from the University of Illinois, UrbanaChampaign, in 1988 and 1990, respectively. Following his graduate studies, he was employed as a Research Scientist at Schlumberger-Doll Research Center, Ridgefield, CT. In 1993 he joined the Electrical and Electronics Engineering Department of Bilkent University, Ankara, Turkey. Since 2006, he is a full professor at Bilkent University. Since 2011, he has been serving as the chairman of department. His current research interests include statistical signal processing, time-frequency analysis and remote sensing. Dr. Arikan has served as chairman of Turkey chapter of IEEE. 